Chapter 4

\title{
Multimode Graded-Index Optical Fibers for Next-Generation Broadband Access
}

\author{
David R. Sánchez Montero and \\ Carmen Vázquez García \\ Additional information is available at the end of the chapter \\ http://dx.doi.org/10.5772/54245
}

\section{Introduction}

Growing research interests are focused on the high-speed telecommunications and data communications networks with increasing demand for accessing to the Internet even from home. For instance, in Nov 2011 Strategy Analytics forecasted that there would be more than 807 million broadband fixed line subscriptions worldwide in 2017, based on a figure of 578 million at the end of 2011 showing a cumulative annual growth rate of around 8 percent [1]. This increasing demand for high-speed information transmission over the last two decades has been driven by the huge successes during the last decade of new multimedia services, commonly referred as Next-Generation Access (NGA) services, such as Internet Protocol Television (IPTV) or Video on Demand (VoD), as well as an increased data traffic driven by High-Definition TV (HDTV) and Peer-to-Peer (P2P) applications which have changed people's habits and their demands for service delivery. Consequently, consumer adoption of broadband access to facilitate use of the Internet for knowledge, commerce and, obviously, entertainment is contingent with the increment of the optical broadband access network capacity, which should extent into the customer's premises up to the terminals. Thus, steady increases in bandwidth requirements of access networks and local area networks (LANs) have created a need for short-reach and medium-reach links supporting data rates of Gbps (such as Gigabit Ethernet, GbE), 10Gbps (such as 10-Gigabit Ethernet, 10GbE) and even higher (such as 40- and 100- Gigabit Ethernet standards, namely 40GbE and $100 \mathrm{GbE}$ respectively, which started in November 2007 and have been very ratified in June 2010). Detailed studies [2,3] have defined the required bit-rates to be transmitted to the customer's premises for different profiles for the traffic flows, reaching a total future-proof 
very-high-bit-rate link in the order of $2 \mathrm{Gbps}$ per user. It is estimated that end-user access bandwidths could reach 1 Gbps by 2015, and 10 Gbps by 2020.

Related to this latter premise, a growing number of service providers are turning to solutions capable of exploiting the full potential of optical fiber for service delivery, being the copper based x-Digital Subscriber Line (xDSL) infrastructure progressively replaced by a fiber-based outside plant with thousands of optical ports and optical fiber branches towards residential and business users, constituting the core of the FTTx (Fiber to the Home/Node/ Curb/Business) deployments, see Fig. 1(a). These include passive optical networks (PONs), whose standardization has accelerated product availability and deployment. The ongoing evolution to deliver Gigabit per second Ethernet and the growing trend to migrate to Wavelength Division Multiplexing (WDM) schemes have benefited significantly from the Coarse WDM (CWDM) and Dense WDM (DWDM) optoelectronics technologies, as they provide a more efficient way to deliver traffic to Customer Premises Equipment (CPE) devices. These systems, commonly referred to as WDM-PON, are still under standardization process and field trials and are the basis of the so-called next-generation broadband optical access networks to prepare for the future upgrade of the FTTx systems currently being deployed. A basic scheme of the WDM-PON architecture is depecited in Fig. 1(b). However, networking architectures such as PON, BPON, WDM-PON, etc. are outside the scope of this chapter. There is a widely-spread consensus concerning service providers that FTTx is the most powerful and future-proof access network architecture for providing broadband services to residential users.

(a)

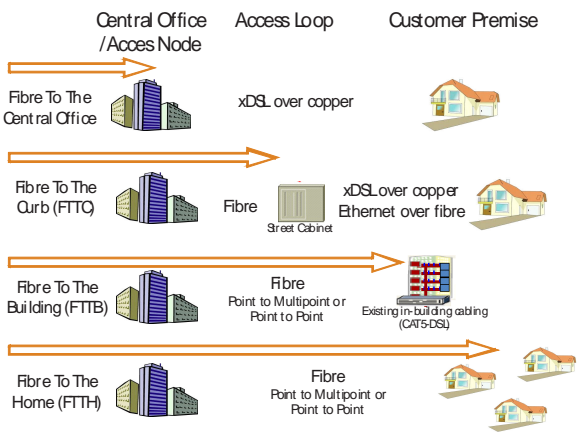

(b)

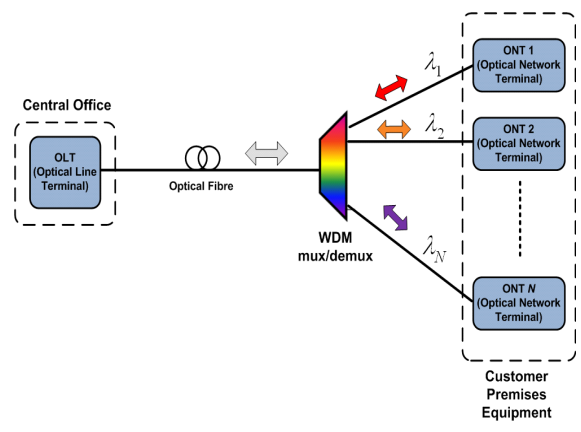

Figure 1. (a) Different FTTx network deployments. (b) Architecture of WDM-PON.

In the FTTx system concepts deployed up to now, singlemode optical fiber (SMF) is used, which has a tremendous bandwidth and thus a huge transport capacity for many services such as the ITU G.983.x ATM1-PON system. Research is ongoing to further extend the capabilities of shared SMF access networks. The installation of SMF has now conquered the core and 
metropolitan area networks and is subsequently penetrating into the access networks. However, it requires great care, delicate high-precision equipment, and highly-skilled personnel, being mainly deployed for long-haul fiber optic communications, constituting the so-called Optical Distribution Network (ODN) and the core telecommunication network of the next generation of optical broadband access networks. Nevertheless, as it comes closer to the end user and his residential area, the costs of installing and maintaining the fiber network become a driving factor, which seriously hampers the large-scale introduction of FTTx.

Also inside the customer's premises, there is a growing need for convergence of the multitude of communication networks. Presently, Unshielded Twisted copper Pair (UTP) cables are used for voice telephony, cat-5 UTP cables for high-speed data, coaxial cables for CATV ${ }^{2}$ and $\mathrm{FM}^{3}$ radio signals distribution, wireless Local Area Network (LAN) for high-speed data, FireWire for high-speed short-range signals, and also Power Line Communications (PLC) technology for control signals and lower-speed data. These different networks are each dedicated and optimised for a particular set of services, which also put different Quality-ofService (QoS) demands, and suffer from serious shortcomings when they are considered to serve the increasing demand for broadband services. Also no cooperation between the networks exists. A common infrastructure that is able to carry all the service types would alleviate these problems. It is therefore not easy to upgrade services, to introduce new ones, nor to create links between services (e.g., between video and data). By establishing a common broadband in-house network infrastructure, in which a variety of services can be integrated, however, these difficulties can be surmounted. The transmission media used at present are not suited for provisioning high-bandwidth services at low cost. For instance, today's wiring in LANs is based mainly on copper cables (twisted pair or coaxial) and silica (glass) fiber of two kinds: singlemode optical fiber (SMF) and multimode optical fiber (MMF). Copper based technologies suffer strong susceptibility to electromagnetic interferences and have limited capacity for digital transmission as well as the presence of crosstalk. Compared to these copper based technologies, optical fiber has smaller volume, it is less bulky and has a smaller weight. In comparison with data transmission capability, optical fiber offers higher bandwidth at longer transmission distances.

On the other hand, optical fiber is extensively used for long-distance data transmission and it represents an alternative for transmission at the customer's premises as well. Optical fiber connections offer complete immunity to EMI and present increase security, since it is very difficult to intercept signals transmitted through the fiber. Moreover, optical communication systems based on silica optical fibers allow communication signals to be transmitted not only over long distances with low attenuation but also at extremely high data rates, or bandwidth capacity. In SMF systems, this capability arises from the propagation of a single optical mode in the low-loss windows of silica located at the near-infrared wavelengths of $1.3 \mu \mathrm{m}$, and $1.55 \mu \mathrm{m}$. Furthermore, since the introduction of Erbium-Doped Fiber Amplifiers (EDFAs), the last decade has witnessed the emergence of SMF as the standard data trans-

2 CATV: Community Antena TeleVision.

3 FM: Frequency Modulation. 
mission medium for wide area networks (WANs), especially in terrestrial and transoceanic communication backbones. The success of the SMF in long-haul communication backbones has given rise to the concept of optical networking, which is a central theme with currently driving research and development activities in the field of photonics. The main objective is to integrate voice, video, and data streams over all-optical systems as communication signals make their way from WANs down to the end user by Fiber-To-The-x (FTTx), offices, and in-homes.

Although conventional SMF solutions have the potential of achieving very large bandwidths, they suffer from high connections costs compared to copper or wireless solutions. For this reason, SMF has not been widely adopted by the end user (premises) where most of the interconnections are needed and less cost-sharing between users is obtainable. The underlying factor is the fact that the SMF core is typically only a few micrometers in diameter with the requirement of precise connecting, delicate installation and handling. Yet as the optical network gets closer to the end user, the system is characterized by numerous connections, splices, and couplings that make the use of thin SMF impractical. An alternative technology is then the use of conventional silica-based multimode optical fiber (MMF) with larger core diameters. This fact allows for easier light coupling from an optical source, large tolerance on axial misalignments, which results in cheaper connectors and associated equipment, as well as less requirements on the skills of the installation personnel. However, the use of MMFs is at a cost of a bandwidth penalty with regards to their SMF counterparts, mainly due to the introduction of modal dispersion. This is the reason why MMF is commonly applied to short-reach and medium-reach applications due to its low intrinsic attenuation despite its limited bandwidth. In particular, in the access network, the use of MMF may yield a considerable reduction of installation costs although the bandwidth-times length product of SMF is significantly higher than that of MMF. As in the access network, the fiber link lengths are less than $10 \mathrm{~km}$, however, the bandwidth of presently commercially available silica MMFs is quite sufficient.

On the other hand, compared to multimode silica optical fiber, polymer optical fiber (POF) offers several advantages over conventional multimode optical fiber over short distances (ranging from $100 \mathrm{~m}$ to $1000 \mathrm{~m}$ ) such as the even potential lower cost associated with its easiness of installation, splicing and connecting. This is due to the fact that POF is more flexible and ductile [4], making it easier to handle. Consequently, POF termination can be realized faster and cheaper than in the case of silica MMF. This POF technology could be used for data transmission in many applications areas ranging like in-home, fiber to the building, wireless LAN backbone or office LAN among others. In addition, improvement in the bandwidth of POF fiber can be obtained by grading the refractive index, thus introducing the socalled Graded-Index POFs (GIPOFs). Although by grading the index profile significantly enhanced characteristics have been obtained, the bandwidth and attenuation still limit the transmission distances and capacity. Reduction of loss has been achieved by using amorphous perfluorinated polymers for the core material. This new type of POF has been named perfluorinated GIPOF (PF GIPOF). This new fiber with low attenuation and large bandwidth has opened the way for high capacity transmission over POF based systems. In addi- 
tion to, as PF GIPOF has a relative low loss wavelength region ranging from 650nm to 1300nm (even theoretically in the third transmission window), it allows for WDM transmission of several data channels. However, attenuation and bandwidth characteristics of the current state-of-the-art PF GIPOF are not at par with those of standard silica SMFs, but they still are superior to those of copper based technologies. Nevertheless, although these losses are coming down steadily due to ongoing improvements in the production processes of this still young technology, the higher than silica attenuation inhibits their use in relative long link applications, being mainly driven for covering in-building optical networks link lengths for in-building/home optical networks (with link lengths less than $1 \mathrm{~km}$ ), and thus the loss per unit length is of less importance. It should be noted that available light sources for silica fiber based systems can be used with PF GIPOF systems. The same is true of connectors as in the case of Gigabit Ethernet equipment.

Therefore, it can be stated that polymer optical fiber technology has reached a level of development where it can successfully replace copper based technology and silica MMF for data transmission in short distance link applications such as in the office, in-home and LAN scenarios. Moreover, PF GIPOF is forecasted to be able to support bit-rate distance products in the order of 10Gbps km [5]. Short distance communications system like in-home network and office LANs represent a unique opportunity for deployment of PF GIPOF based systems for broadband applications. We can conclude that PF GIPOF technology is experiencing rapid development towards a mature solution for data transmission at short haul communications. The challenge remains in bringing this POF technology (transceiver, connectors,...) to a competitive price and performance level at the customer's premises.

Nevertheless, the potentials of these multimode fibers, both silica- and polymer-based, to support broadband radio-frequency, microwave and even millimetre wave transmission over short- and medium-reach distances are yet to be fully known. The belief is that a better understanding of the factors that affect the fiber bandwidth will prove very useful in increasing the bandwidth of silica MMF and PF GIPOF links in real situations. In the whole fiber network society to be realized in the near future, it is said that silica-based SMF fibers for long-haul backbone will be only several percents of the total use, and the remaining more than $90 \%$ would correspond to all-optical networks covering the last mile [6]. Link lengths may range from well below $1 \mathrm{~km}$ in LANs and residential houses, to only a few kilometres in larger building such as offices, hospitals, airport halls, etc. And it is now clear that the expected market is huge and researches and companies all over the world are competing to find a solution to this issue.

In this framework, the first part of this chapter, comprising sections 2 and 3 will briefly address the fundamentals of mutimode optical fibers as well as present transmission capacities. Like any communication channel, the multimode optical fiber also suffers from various signal distortions limiting its usefulness. The primary mechanisms contributing to the channel impairment in multimode fibers are discussed. Both silica-based MMFs and PF GIPOFs are essentially large-core optical waveguide supporting multiple transverse electromagnetic modes and they suffer from similar channel impairments. On the other hand, present capabilities of actual multimode optical fiber-based deployments are shown. In addition, different techni- 
ques reported in literature to carry microwave and millimetre-wave over optical networks, surmounting the multimode fiber bandwidth bottleneck, are also briefly described.

The second part of this chapter, which comprises sections 4, 5 and 6, respectively, focuses on the frequency response mathematical framework and the experimental results, respectively, of both types of multimode optical fibers. Some of the key factors affecting the frequency characteristics of both fiber types are addressed and studied. Theoretical simulations and measurements are shown for standard silica-based MMF as well as for PF GIPOF. Although some of these issues are interrelated, they are separately identified for clarity.

Finally, the main conclusions of this chapter are reported in Section 7.

\section{Fundamentals of multimode optical fibers}

Despite the above advantages, the use of multimode optical fiber has been resisted for some years by fiber-optic link designers in favour of their SMF counterparts since Epworth discovered the potentially catastrophic problem of modal noise [7]. Modal noise in laser-based MMF links has been recently more completely addressed and theoretical as well as experimental proofs have shown that long-wavelength operation of MMFs is robust to modal noise [8-10]. This explains the spectacular regain of interest for MMFs as the best solution for the cabling of the access, in-home networks and LANs. The question that needs answer now in view of increasing the usefulness of MMF concerns the improvement of their dispersion characteristics, which is related to their reduced bandwidth.

For the transmission of communication signals, attenuation and bandwidth are important parameters. Both parameters will be briefly described in the following subsections, focusing on their impact over multimode fibers. In any case, the optical signal is distorted and attenuated when it propagates over the fiber. These effects have to be modeled when describing the signal transmission. They behave quite differently in different types of fibers. Whereas signal distortions in singlemode fibers (SMFs) are primarily caused by chromatic dispersion, i.e. the different speeds of individual spectral parts, the description of dispersion in multimode fibers (MMFs) is considerably more complex. Not only does chromatic dispersion occur in them, but also has the generally much greater modal (or intermodal) dispersion.

It should be noted that, apart from attenuation, an important characteristic of an optical fiber as a transmission medium is its bandwidth. Bandwidth is a measure of the transmission capacity of a fiber data link. As multimode fibers can guide many modes having different velocities, they produce a signal response inferior to that of SMFs, being this modal dispersion effect the limiting bandwidth factor. So bandwidth and dispersion are two parameters closely related.

\subsection{Attenuation}

Attenuation in fiber optics, also known as transmission loss, is the reduction in the intensity of the light beam with respect to distance traveled through a transmission medium, being an 
important factor limiting the transmission of a digital signal across large distances. The attenuation coefficient usually use units of $\mathrm{dB} / \mathrm{km}$ through the medium due to the relatively high quality of transparency of modern optical transmission media. Empirical research over the years has shown that attenuation in optical fiber is caused primarily by both scattering and absorption. However, the fundamentals of both attenuation mechanisms are outside the scope of this chapter.

On the one hand, silica exhibits fairly good optical transmission over a wide range of wavelengths. In the near-infrared (near IR) portion of the spectrum, particularly around $1.5 \mu \mathrm{m}$, silica can have extremely low absorption and scattering losses of the order of $0.2 \mathrm{~dB} / \mathrm{km}$. Such remarkably-low losses are possible only because ultra-pure silicon is available, being essential for manufacturing integrated circuits and discrete transistors. Nevertheless, fiber cores are usually doped with various materials with the aim of raising the core refractive index thus achieving propagation of light inside the fiber (by means of total internal reflection mechanisms). A high transparency in the 1.4- $\mu$ m region is achieved by maintaining a low concentration of hydroxyl groups $(\mathrm{OH})$. Alternatively, a high $\mathrm{OH}$ concentration is better for transmission in the ultraviolet (UV) region.

On the other hand, until recently, the only commercially available types of POF were based on non-fluorinated polymers such as PolyMethylMethAcrylate (PMMA) (better known as Plexiglass $\left.{ }^{\circledR}\right)$, widely used as core material for graded-index fiber [11] in addition with the utilization of several kinds of dopants. Although firstly developed PMMA-GIPOFs were demonstrated to obtain very high transmission bandwidth compared to that of Step-Index (SI) counterparts, the use of PMMA is not attractive due to its strong absorption driving a serious problem in the PMMA-based POFs at the near-IR (near-infrared) to IR regions. This is because of the large attenuation due to the high harmonic absorption loss by carbon-hydrogen (C-H) vibration (C-H overtone). As a result, PMMA-based POFs could only be used at a few wavelengths in the visible portion of the spectrum, typically $530 \mathrm{~nm}$ and $650 \mathrm{~nm}$, with typical attenuations around $150 \mathrm{~dB} / \mathrm{km}$ at $650 \mathrm{~nm}$. Today, unfortunately, almost all gigabit optical sources operate in the near-infrared (typically $850 \mathrm{~nm}$ or $1300 \mathrm{~nm}$ ), where PMMA and similar polymers are essentially opaque. Nevertheless, in this scenario, undistorted bit streams of $2.5 \mathrm{Gbps}$ over $200 \mathrm{~m}$ of transmission length were successfully demonstrated over PMMA-GIPOF [12].

On the other hand, it has been reported that one can eliminate this absorption loss by substituting the hydrogen atoms in the polymer molecule for heavier atoms [13]. In this case, if the absorption loss decreases with the substitution of hydrogen for deuterium or halogen atoms (such as fluorine), the possible distance for signal transmission would be limited by dispersion, and not by attenuation. Many polymers have been researched and reported in literature in order to improve the bandwidth performance given by the first PMMA-based graded-index polymer optical fibers [14]. Nevertheless, today, amorphous perfluorinated (PF) GIPOF is widely used because of its high bandwidth and low attenuation from the visible to the near IR wavelengths compared to PMMA GIPOF [15]. As a result, it is immediately compatible with gigabit transmission sources, and can be used over distances of hundreds of meters. This fact is achieved mainly by reducing the number of carbon-hydro- 
gen bonds that exist in the monomer unit by using partially fluorinated polymers. In 1998, the PF-based GIPOF had an attenuation of around $30 \mathrm{~dB} / \mathrm{km}$ at $1310 \mathrm{~nm}$. Attenuation of $15 \mathrm{~dB} / \mathrm{km}$ was achieved only three years after and lower and lower values of attenuation are being achieved. The theoretical limit of PF-based GIPOFs is $\sim 0.5 \mathrm{~dB} / \mathrm{km}$ at $1250-1390 \mathrm{~nm}$ [16]. In the estimation, the attenuation factors are divided in two: material-inherent scattering loss and material-inherent absorption loss. The first factor is mainly given by the Rayleigh scattering, following the relation $\alpha_{R} \sim(\lambda)^{-4}$. The second factor is given by the absorption caused by molecular vibrations. A detailed explanation on the estimation processes is described [17].

\subsection{Dispersion}

As aforementioned, pulse broadening in MMFs is generally caused by modal dispersion and chromatic dispersion. For MMFs it is necessary to consider the factors of material, modal and profile dispersion. The latter considers the wavelength dependence on the relative refractive index difference in graded index fibers. Waveguide dispersion additionally occurs in singlemode fibers, whereas profile dispersion and modal dispersion do not.

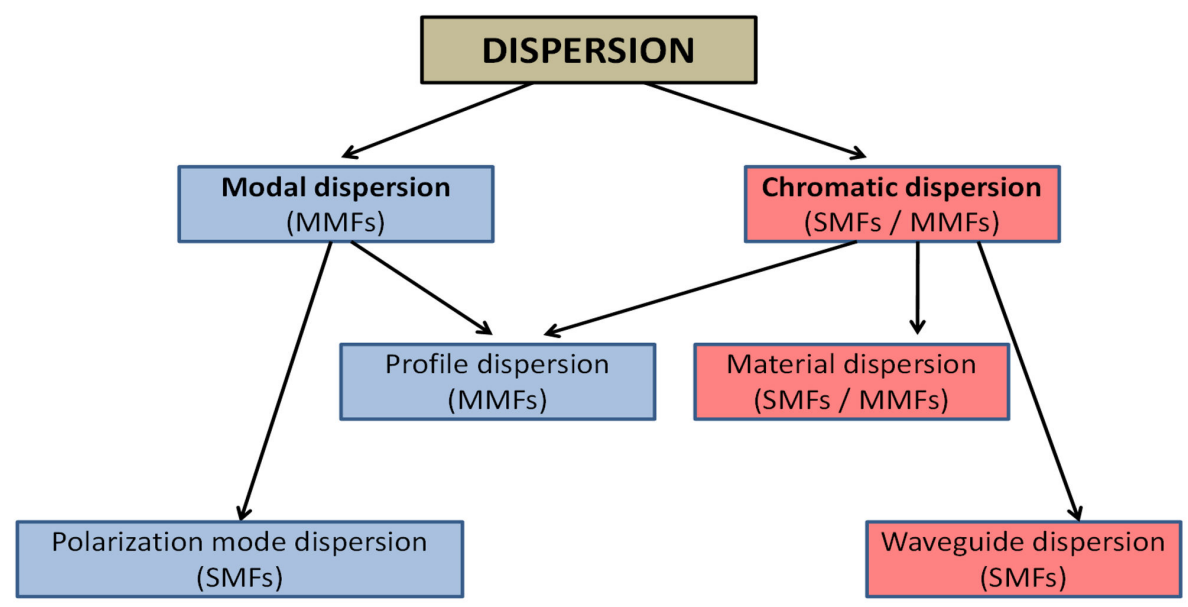

Figure 2. Dispersion mechanisms in optical fibers.

All the kinds of dispersion appearing in optical fibers are summarized in Fig. 2. The mechanisms dependent on the propagation paths are marked in blue, whereas the wavelength-dependent processes are marked in red. Those mechanisms only affecting SMFs are outside from the scope of this work so they will be avoided. For multimode fibers modal dispersion and chromatic dispersion are the relevant processes to be considered.

In a generic description, chromatic dispersion is introduced by the effect that the speed of propagation of light of different wavelengths differs resulting in a wavelength dependence of the modal group velocity. The end result is that different spectral components arrive at 
slightly different times, leading to a wavelength-dependent pulse spreading, i.e. dispersion. As a matter of fact, the broader the spectral width (linewidth) of the optical source the greater is the chromatic dispersion. In PF-based POFs the chromatic dispersion is much smaller than in silica MMF for wavelengths up to $1100 \mathrm{~nm}$. For wavelengths above $1100 \mathrm{~nm}$, the dispersion of the PF-based GIPOF retains and the dispersion of silica MMF increases. The expression of such dispersion is given by:

$$
\Delta t_{\text {chrom }}=D(\lambda) \cdot \Delta \lambda \cdot L ; D(\lambda)=-\frac{\lambda}{c} \cdot \frac{d^{2} n(\lambda)}{d \lambda^{2}}
$$

where $D(\lambda)$ is the material dispersion parameter (usually given in $\mathrm{ps} / \mathrm{nm} \cdot \mathrm{km}$ ), $\Delta \lambda$ is the spectral width of the light source, and $L$ is the length of the fiber. Fig. 3(a) depicts a typical material dispersion curve as a function of the operating wavelength. for a PF GIPOF as well as a silica-based MMF with a $\mathrm{SiO}_{2}$ core doped with $6.3 \mathrm{~mol}-\% \mathrm{GeO}_{2}$ and $\mathrm{SiO}_{2}$ cladding. It is clearly seen the better performance in terms of material dispersion of the PF GIPOF compared to the silica-based counterpart, especially in the range up to $1100 \mathrm{~nm}$.

(a)

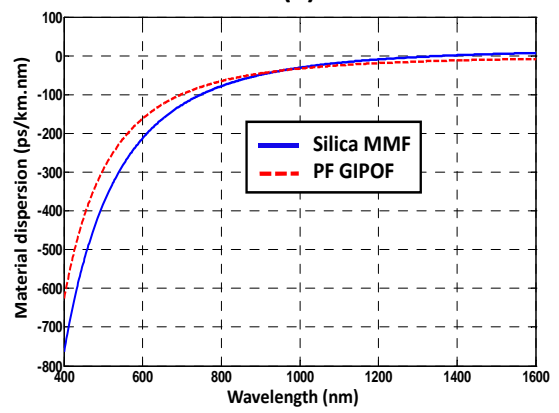

(b)

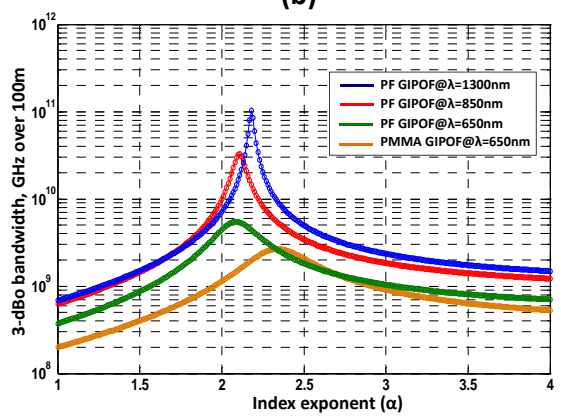

Figure 3. (a) Typical material dispersion of the central core region for a silica-based MMF (blue solid line) and PF GIPOF (red dashed line). (b) Relation between the refractive index profile and bandwidth of 100m-long PF GIPOF. PMMAGIPOF at 650nm is plotted for comparison.

On the other hand, modal dispersion is caused by the fact that the different modes (light paths) within the fiber carry components of the signals at different velocities, which ultimate results in pulse overlap and a garbled communications signal. Lower order modes propagate mainly along the waveguide axis, while the higher-order modes follow a more zigzag path, which is longer. If a short light pulse is excited at the input of the fiber, the lowest order modes arrive first at the end of the fiber and the higher order modes arrive later. The output pulse will thus be built up of all modes, with different arrival times, so the pulse is broadened. 
To overcome and compensate for modal dispersion, the refractive index of the fiber core (or, alternatively, graded index exponent of the fiber core) is graded parabola-like from a high index at the fiber core center to a low index in the outer core region, i.e. by forming a graded-index (GI) fiber core profile. In such fibers, light travelling in a low refractive-index structure has a higher speed than light travelling in a high index structure and the higher order modes bend gradually towards the fiber axis in a shorter period of time because the refractive index is lower at regions away from the fiber core. The objective of the GI profile is to equalise the propagation times of the various propagating modes. Therefore, the time difference between the lower order modes and the higher order modes is smaller, and so the broadening of the pulse leaving the fiber is reduced and, consequently, the transmission bandwidth can be increased over the same transmission length. For negligible modal dispersion the ideal refractive index profile is around 2. This refractive index profile formed in the core region of multimode optical fibers plays a great role determining its bandwidth, because modal dispersion is generally dominant in the multimode fiber although an optimum refractive index profile can produce the minimum modal dispersion, i.e. larger bandwidth being almost independent of the launching conditions [18]. Fig. 3(b) shows the calculated bandwidth of a PF-based GIPOF operating at different wavelengths, in which it is assumed that the source spectral width is $1 \mathrm{~nm}$, with regards to the refractive index profile, $\alpha$. The data of the bandwidth of a PMMA-based GIPOF at $650 \mathrm{~nm}$ is also shown for comparison showing a maximum limited to approximately $1.8 \mathrm{GHz}$ for $100 \mathrm{~m}$ by the large material dispersion. On the other hand, the smaller material dispersion of the PF polymer-based GIPOF permits a maximum bandwidth of $4 \mathrm{GHz}$ even at $650 \mathrm{~nm}$. Furthermore, when the signal wavelength is $1300 \mathrm{~nm}$, theoretical maximum bandwidth achieves $92 \mathrm{GHz}$ for $100 \mathrm{~m}$. The difference of the optimum index exponent value between $650 \mathrm{~nm}$ and $1300 \mathrm{~nm}$ wavelengths is caused by the inherent polarization properties of material itself. It should be mentioned that a uniform excitation has been assumed and no differential mode attenuation (DMA) and mode coupling (MC) effects have been considered. These effects will be briefly described later on.

To summarize, the different types of dispersion that appear in a MMF and their relation to the fiber bandwidth are analyzed in Fig. 4. This figure reports the PF GIPOF chromatic and modal dispersion and the total bandwidth of a 100m-long link as a function of the refractive index profile, at a wavelength of 1300nm. Fig. 4(b) depicts the corresponding 3-dBo (3-dB optical bandwidth) baseband bandwidth, related to Fig. 4(a). These plots are based on the same analysis of Fig. 3, which assumed a uniform excitation and neglected both the DMA and mode coupling effects. From these figures, the chromatic bandwidth is seen to show little dependence on $\alpha$, which means that the material dispersion is the dominant contribution (with regards to the profile dispersion) in the transmission window considered. On the other hand, the modal bandwidth shows a highly peaked resonance with $\alpha$. This is the well known characteristic feature of the grading. With the present choice of parameters values, that maximum bandwidth (i.e. minimum dispersion) approximately occurs at 2.18 at 1300nm, as shown in Fig. 4(a). Furthermore, the presence of crossover points (namely $\alpha_{1}$ and $\alpha_{2}$ ) shows that the total bandwidth may be limited either by the modal dispersion or the chromatic dispersion depending on the value of the refractive index profile. Focusing on 
Fig. 4(a), the chromatic dispersion will essentially limit the total bandwidth for $\alpha_{1}<\alpha<\alpha_{2}$, whilst for $\alpha<\alpha_{1}$ or $\alpha>\alpha_{2}$ the modal dispersion will cause the main limitation. In other words, when the index exponent is around the optimum value ( $\alpha$-resonance), the modal dispersion effect on the possible 3-dB bandwidth (and so on the bit rate) is minimized and the chromatic dispersion dominates this performance. On the other hand, when the index exponent is deviated from the optimum, the modal dispersion increases becoming the main source of bandwidth limitation.

(a)

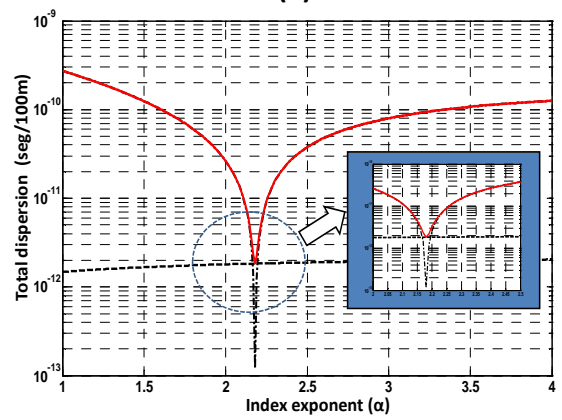

(b)

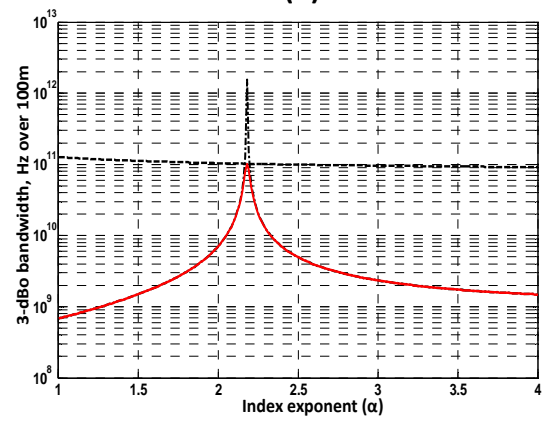

Figure 4. (a) Dispersion effects versus refractive index profile for a 100m-long PF GIPOF, assuming equal power in all modes and a $1300 \mathrm{~nm}$ light source with $1 \mathrm{~nm}$ of spectral linewidth. Inset: zoom near the optimum profile region. (b) Corresponding 3-dBo bandwidth. (-) Total dispersion ; (- -) Modal dispersion ; (---) Chromatic dispersion.

It is also noteworthy that, since the PF polymer has low material and profile dispersions and the wavelength dependence of the optimum profile is decreased, a high bandwidth performance can be maintained over a wide wavelength range, compared to multimode silica or PMMA-based GIPOF fibers.

\subsubsection{Dispersion modelling approach}

The propagation characteristics of optical fibers are generally described by the wave equation which results directly from Maxwell's equations and characterizes the wave propagation in a fiber as a dielectric wave guide in the form of a differential equation. In order to solve the equation, the field distributions of all modes and the attendant propagation constants, which results from the use of the boundary conditions, have to be determined.

The wave equation is basically a vector differential equation which can, however, under the condition of weak wave guidance be transformed into a scalar wave equation in which the polarization of the wave plays no role whatsoever [19]. The prerequisite for the weak wave guiding is that the refractive indices between the core and cladding hardly differ, being fulfilled quite well in silica fibers when the difference in refractive index between the core and cladding region is below $1 \%$. Calculations based on the scalar wave equation only show very small inaccuracies with regards to the group delay. Then, the equations which describe the electric and magnetic fields are decoupled so that you can write a scalar wave equation. 
The models based on the solution of the wave equation in the form of a mode solver differ fundamentally only in regard to the solution method and whether or not you are proceeding from a more computer-intensive vector wave equation or the more usual scalar wave equation. In the technical literature solutions for the vector wave equation with the aid of finite element method (FEM) [20], with finite differences (Finite Difference Time Domain Method FDTD) [21] and the beam propagation method (BPM) [22] are well known. These are generally used for very small, mostly singlemode waveguides in which polarization characteristics play a role. Multimode fibers (including polymer fibers) are quite large and the polarization of light counts for only a few centimeters. That is why analytical estimations of the scalar wave equation, the so-called WKB (Wentzel-Kramers-Brillouin, from whom the name derives) Method and Ray Tracing [23], are primarily used for the modeling of multimode fibers. In the latter, the propagating light through an optical system can be seen as the propagation of individual light rays following a slightly different path; these paths can be calculated using standard geometrical optics.

Focusing on the WKB method, the latter primarily makes available expressions, that can be calculated efficiently, for describing the propagations constants and group delays of the propagating modes within the fiber. In this method, whereas the field distributions in step index profile fibers can be determined analytically, the refractive index distribution over the radius of a graded index fiber can generally be described with a power-law, as Eq. 2 states. Fibers with power-law profiles possess the characteristic that the modes can be put in mode groups which have the same propagation constant and also similar mode delay (at least for exponents close to $\alpha=2$ ). The propagation times of the modes are only then dependent on the propagation constant and then the group delay can be determined with the aid of the WKB Method by differentiating the propagation constant from the angular frequency [24].

$$
n(r, \lambda)= \begin{cases}n_{1}(\lambda)\left[1-2 \Delta(\lambda)\left(\frac{r}{a}\right)^{\alpha}\right]^{1 / 2} & \text { for } 0 \leq \mathrm{r} \leq \mathrm{a} \\ n_{1}(\lambda)[1-2 \Delta(\lambda)]^{1 / 2} & \text { for } \mathrm{r} \geq \mathrm{a}\end{cases}
$$

where $r$ is the offset distance from the core center, $a$ is the fiber core radius (i.e. the radius at which the index $n(r, \lambda)$ reaches the cladding value $\left.n_{2}(\lambda)=n_{1}(\lambda)[1-2 \Delta(\lambda)]^{1 / 2}\right), n_{1}(\lambda)$ is the refractive index in the fiber core center, $\lambda$ is the free space wavelength of the fiber excitation light, $\alpha$ is the refractive index exponent and $\Delta(\lambda)$ is the relative refractive index difference between the core and the cladding. It is usually assumed that the core and cladding refractive index materials follow a three-term Sellmeier function of wavelength [25] given by:

$$
n_{i}(\lambda)=\left(1+\sum_{k=1}^{3} \frac{A_{i, k} \lambda^{2}}{\lambda^{2}-\lambda_{i, k}^{2}}\right)^{1 / 2} \quad \text { with i=1 (core), } 2 \text { (cladding) }
$$


where $A_{i, k}$ and $\lambda_{i, k}$ are the oscillator strength and the oscillator wavelength, respectively (both parameters are often gathered under the term of Sellmeier constants).

On the other hand, from the WKB analysis, the modal propagation constants can be approximately derived as following [26], in which each guided mode has its own propagation constant and therefore propagates at its own particular velocity:

$$
\beta_{m}=\beta(m, \lambda)=n_{1}(\lambda) k\left[1-2 \Delta(\lambda)\left(\frac{m}{M(\alpha, \lambda)}\right)^{\frac{2 \alpha}{\alpha+2}}\right]^{1 / 2}
$$

where $m$ stands for the principal mode number [27] and $k=2 \pi / \lambda$ is the free space wavenumber. This so-called principal mode number (mode group number or mode number) can be defined as $m=2 \mu+v+1$ in which the parameters $\mu$ and $v$ are referred to as radial and azimuthal mode number, respectively. Physically, $\mu$ and $v$ represent the maximum intensities that may appear in the radial and azimuthal direction in the field intensities of a given mode. For a deeper analysis works reported in [28, 29] are recommended. On the other hand, $M(\alpha, \lambda)$ is the total number of mode groups that can be potentially guided in the fiber, given by [26]:

$$
M(\alpha, \lambda)=2 \pi a \frac{n_{1}(\lambda)}{\lambda}\left[\frac{\alpha \cdot \Delta(\lambda)}{\alpha+2}\right]^{1 / 2}
$$

As a consequence of Eq. 4, the delay time $\tau(m, \lambda)$ of a mode depends only on its principal mode number. It should be mentioned that the differences in modal delay are those that determine the modal dispersion. The delay time of the guided modes (or modal delay per unit length) can be derived from Eq. 4 using the definition:

$$
\tau(m, \lambda)=-\frac{\lambda^{2}}{2 \pi c} \frac{d \beta(m, \lambda)}{d \lambda}
$$

where $c$ is the speed of light in vacuum, deriving in:

$$
\tau_{m}=\tau(m, \lambda)=\frac{N_{1}(\lambda)}{c}\left[1-\frac{\Delta(\lambda)(4+\varepsilon(\lambda))}{\alpha+2}\left(\frac{m}{M}\right)^{\frac{2 \alpha}{\alpha+2}}\right]\left[1-2 \Delta(\lambda)\left(\frac{m}{M}\right)^{\frac{2 \alpha}{\alpha+2}}\right]^{-1 / 2}
$$

where $\varepsilon(\lambda)$ is the profile dispersion parameter given by [30]: 


$$
\varepsilon(\lambda)=-\frac{2 n_{1}(\lambda)}{N_{1}(\lambda)} \frac{\lambda \frac{d \Delta(\lambda)}{d \lambda}}{\Delta(\lambda)}
$$

and $N_{1}(\lambda)$ is the material group index defined by:

$$
N_{1}(\lambda)=n_{1}(\lambda)-\lambda \frac{d n_{1}(\lambda)}{d \lambda}
$$

\subsection{Differential mode attenuation}

The distribution of the power among the different modes propagating through the fiber will also be affected by the Differential Mode Attenuation (DMA), also called mode-dependent attenuation, which causes the attenuation coefficient to vary from mode to mode in a different manner. It originates from conventional loss mechanisms that are present in usual optical fibers such as absorption, Rayleigh scattering [31] or losses on reflection at the corecladding interface [32]. The following functional expression or empirical formula for the DMA is proposed, in which the DMA increases when incresing the mode order [33]:

$$
\alpha_{m}=\alpha_{m}(m, \lambda)=\alpha_{o}(\lambda)+\alpha_{o}(\lambda) I_{\rho}\left[\eta\left(\frac{m-1}{M}\right)^{\frac{2 \alpha}{\alpha+2}}\right]
$$

where $\alpha_{o}(\lambda)$ is the attenuation of low-order modes (i.e intrinsic fiber attenuation), $I_{\rho}$ is the Qth order modified Bessel function of the first kind and $\eta$ is a weighting constant. This empirical formula is set up by noticing that most measured DMA data displayed in the literature for long wavelengths conform to the shape of modified Bessel functions [31, 34, 35]. It is also worth mentioning that, during propagation, modes with fastest power loss may be stripped off or attenuated so strongly that they no longer significantly contribute to the dispersion. In other words, the DMA is a filtering effect, which may yield a certain bandwidth enhancement depending on the launching conditions and the transmission length. From Fig. 5 it can be seen that low-order mode groups show similar attenuation (intrinsic fiber attenuation) whereas for high-order mode groups attenuation increases rapidly.

\subsection{Mode coupling}

Mode coupling is rather a statistical process in which modes exchange power with each other. Due to the mode coupling, the optical energy of the low-order modes would be coupled to higher-order modes, even if only the low-order modes would have launched selectively. This effect generally occurs through irregularities in the fiber, whether they are roughness of the core-cladding interface or impurities in the core material leading, for instance, to refrac- 
(a)

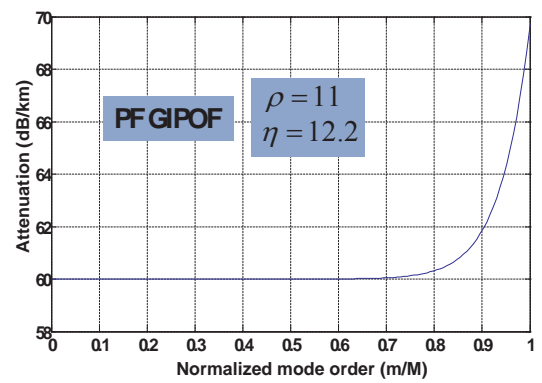

(b)

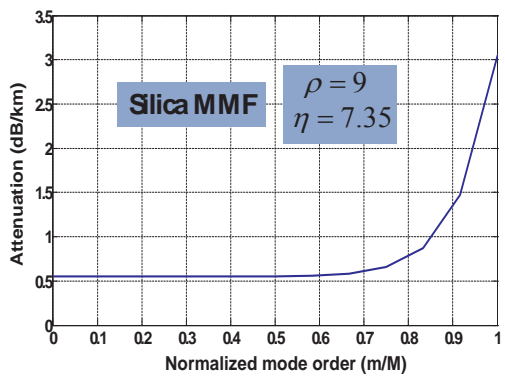

Figure 5. (a) Differential mode attenuation (DMA) as a function of the normalized mode order m/M for a PF GIPOF with $a=250 \mu \mathrm{m}, a=2$, and $\lambda=1300 \mathrm{~nm}$. An intrinsic attenuation of $60 \mathrm{~dB} / \mathrm{km} @ 1300 \mathrm{~nm}$ has been considered. (b) Differential mode attenuation (DMA) as a function of the normalized mode order $\mathrm{m} / \mathrm{M}$ for a silica MMF with $\mathrm{a}=31.25 \mu \mathrm{m}$, $\mathrm{a}=2$, and $\lambda=1300 \mathrm{~nm}$. An intrinsic attenuation of $0.55 \mathrm{~dB} / \mathrm{km} @ 1300 \mathrm{~nm}$ has been considered.

tive index fluctuations. This effect can therefore only be described with statistical means. In addition, it is agreed that silica-based MMFs exhibit far less mode coupling compared to POF fibers [36]. This is attributed to the difference in the material properties.

The main effects for generating mode coupling are Rayleigh and Mie scattering which differ in the size of the scattering centers. Rayleigh scattering arises through the molecular structure of matter which is why no material can have perfectly homogenous properties. Its optical density fluctuates around a mean value which represents the refractive index of the material. These fluctuations are very small and have typical sizes in the range of molecules $(<\mu \mathrm{m})$. Rayleigh scattering depends on the wavelength and decreases with greater wavelengths as of the fourth power $\left(\sim \lambda^{-4}\right)$. In constrast, Mie scattering comes from the fluctuations of the refractive index which has greater typical lengths that mostly come about because of impurities in the material such as air bubbles or specks of dust which are large compared with the wavelength of light. The ensuing scattering has more of an effect on the direction of propagation of the light and is independent of the wavelength. Thinking of these aspects mode coupling reveals itself as a complex process which plays a great role in polymer fibers.

There are some approaches for the modeling of mode coupling which cannot be applied equally well in all propagation models $[37,38]$ while some descriptions present themselves rather in mode models [39]. Moreover, the coupling coefficients which describe the coupling between modes can either be described by analytical attempts which are based on observations of mode overlapping [40,41]. However, it is demonstrated that in real fibers only very few modes effectively interact with each other and, moreover, neighboring or adjacent modes (those with similar propagation constants, modes $m$ and $m \pm 1$, respectively) primarily show strong mode coupling [42,43]. As a matter of fact, larger core refractive index and higher fiber numerical aperture (NA) values are expected to decrease the mode coupling in GIPOFs. In addition, larger mode coupling effects are observed in SIPOFs compared to that GIPOFs counterparts. 
Mode coupling alters the achievable bandwidth of a multimode fiber. According to the laws of statistics, the differential delay (or more precisely, the standard deviation) between the different propagating modes does not increase in a linear relationship to the length but approximately only proportional to the square root of the length. The best known approach for approximately determining the coupling length of the fiber is the description with the aid of a length-dependent bandwidth, in the way $B W \propto L \gamma$. Here the coupling length is the point in which the linear decrease $(\gamma \approx-1)$ in the bandwidth turns to a root dependency $(\gamma \approx-0.5)$ under mode coupling. From this point, a state of equilibrium arises through mode coupling effects. Typical values of coupling length in silica-based GI-MMFs are in the order of units of kilometers [44] whereas in the case of PF GIPOFs usually range from 50m up to $150 \mathrm{~m}$.

\section{Multimode optical fiber capabilities}

Emerging themes in next-generation access (NGA) research include convergence technologies, in which wireline-wireless convergence is addressed by Radio-over-Fiber (RoF) technologies. Photonics will transport gigabit data across the access network, but the final link to the end-user (measured in distances of metres, rather than $\mathrm{km}$ 's) could well be wireless, with portable/mobile devices converging with photonics. RoF technologies can address the predicted multi-Gbps data wave, whilst conforming to reduced carbon footprints (i.e. green telecoms). NGA networks will provide a common resource, with passive optical networks (PONs) supplying bandwidth to buildings, and offering optical backhaul for such systems.

\begin{tabular}{ll}
\hline Parameter & Remarks \\
\hline Transmission distances & Typ. $<10 \mathrm{~km}$, max. $20 \mathrm{~km}$, e.g. for alternative topologies \\
\hline Peak data rate & $100 \mathrm{Mbps}$ (private customers) \\
& $\mathrm{Nx} 1 \mathrm{Gbps}$ up to $10 \mathrm{Gbps}$ (business) \\
\hline Temperature range & Controlled: $+10^{\circ} \mathrm{C}$ to $+50^{\circ} \mathrm{C}$ \\
& Uncontrolled operation in buildings: $-5^{\circ} \mathrm{C}$ to $+85^{\circ} \mathrm{C}$ \\
& Uncontrolled operation in the field: $-33^{\circ} \mathrm{C}$ to $+85^{\circ} \mathrm{C}$ \\
\hline
\end{tabular}

Long lifetime

Humidity and vibrations (shock) have to be considered at non-weather protected locations

No optical amplifiers in the field

No optical dispersion compensation

Table 1. Access network requirements.

It has been indicated by several roadmaps that the peak link data rate should be at least $100 \mathrm{Mbps}$ (symmetrical) for private customers and 1 to $10 \mathrm{Gbps}$ for business applications. Inherent access network requirements are highlighted in Table 1. These hundreds of megabits per second per user are reasonably reachable in the coming future and the Fiber To The 
Home (FTTH, or some intermediate version such as FTT-curb) network constitutes a fiber access network, connecting a large number of end users to a central point, commonly known as an access node. Each access node will contain the required active transmission equipment used to provide the applications and services over optical fiber to the subscriber.

On the one hand, Ethernet is the most widespread wired LAN technology, including inhome networks, and the development of Ethernet standards goes hand in hand with the adoption and development of improved MMF channels [45]. And Ethernet standards for $1 \mathrm{Gbps}$ and 10Gbps designed for multimode and singlemode fibers are now in use. Table 2 shows the minimum performance specified by IEEE 802.3 standard for the various interfaces. For example, 10-Gigabit Ethernet (GbE) standard operating at 10.3125Gbps@1300nm supports a range of transmission lengths of $300 \mathrm{~m}$ over multimode silica fiber and $10 \mathrm{~km}$ over singlemode silica fiber. Actually OM4 fiber type is under consideration although is not yet

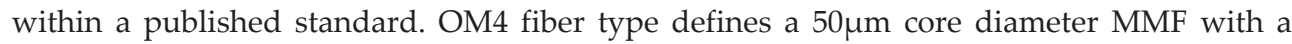
minimum modal bandwidth (under OverFilled Launching condition, OFL) of $3500 \mathrm{MHz}$. km@850nm and 500MHz·km@1300nm, respectively. Nevertheless, data rate transmission research achievements are not at par as those covered by the standard and report even greater values. Some significant works are reported in [46-48]. Different techniques or even a combination of some of them were applied to achieved these transmission records. Some of them will be briefly discussed in next section.

\begin{tabular}{|c|c|c|c|}
\hline \multirow{5}{*}{ Fiber type } & \multirow{5}{*}{$\begin{array}{c}\text { 10GBaseSR } \\
\text { 850nm } \\
\text { Modal Bandwidth / } \\
\text { Operating Range } \\
(\mathrm{MHz} \cdot \mathrm{km}) /(\text { Meters })\end{array}$} & \multirow{5}{*}{$\begin{array}{l}\text { 10GBaseLR(/ER) } \\
\text { 850nm } \\
\text { Modal Bandwidth / } \\
\text { Operating Range } \\
(\mathrm{MHz} \cdot \mathrm{km}) /(\mathrm{Km})\end{array}$} & \multirow{5}{*}{$\begin{array}{c}\text { 10GBaseLRM } \\
\text { 1300nm } \\
\text { Modal Bandwidth /Operating } \\
\text { Range } \\
(\mathrm{MHz} \cdot \mathrm{km}) /(\text { Meters })\end{array}$} \\
\hline & & & \\
\hline & & & \\
\hline & & & \\
\hline & & & \\
\hline $62.5 \mu \mathrm{m}^{*}$ & $160 / 26$ & n.a. & n.a. \\
\hline $62.5 \mu \mathrm{m}(\mathrm{OM}-1)^{* *}$ & $200 / 33$ & n.a. & $500 / 300$ \\
\hline $50 \mu \mathrm{m}$ & $400 / 66$ & n.a. & $400 / 240$ \\
\hline $50 \mu \mathrm{m}(\mathrm{OM}-2)$ & $500 / 82$ & n.a. & $500 / 300$ \\
\hline $50 \mu \mathrm{m}(\mathrm{OM}-3)$ & $2000 / 300$. & n.a. & $500 / 300$ \\
\hline SMF & n.a. ${ }^{* * *}$ & $10(/ 40)$ & n.a/10000. \\
\hline \multicolumn{4}{|c|}{$\begin{array}{l}\text { TIA (Telecommunications Industry Association), Document 492AAAA compliance. Commonly referred to as 'FDDI- } \\
\text { grade' fiber. }\end{array}$} \\
\hline \multicolumn{4}{|c|}{ ** ISO (International Standards Organization), Document 11801 compliance. } \\
\hline *** n.a.: not available. & & & \\
\hline
\end{tabular}

Table 2. 10-Gigabit Ethernet transmission over fiber standards (IEEE 802.3aq). Approved in 2006.

Figure 6 provides a brief description of the current 10GbE and the possible future Ethernet standards over copper and fiber links [6]. The trend of extending the reach and data rate of 
the links is obvious in the previous standards and the $10 \mathrm{GbE}$ standards shown in the figure. Although the twisted pair of copper wires is a relatively low-cost and low-power solution compared to the MMF solutions, the motivation for the transition from the copper-based links to the MMF links is their much higher available bandwidth. However, the need for even higher performance MMF solutions is apparent, and much more is to be expected, for example, with new ultra-HDTV format such as $4 \mathrm{~K}$ (4000 horizontal pixels, with an expected increase in the required bandwidth of a factor of approximately 16).

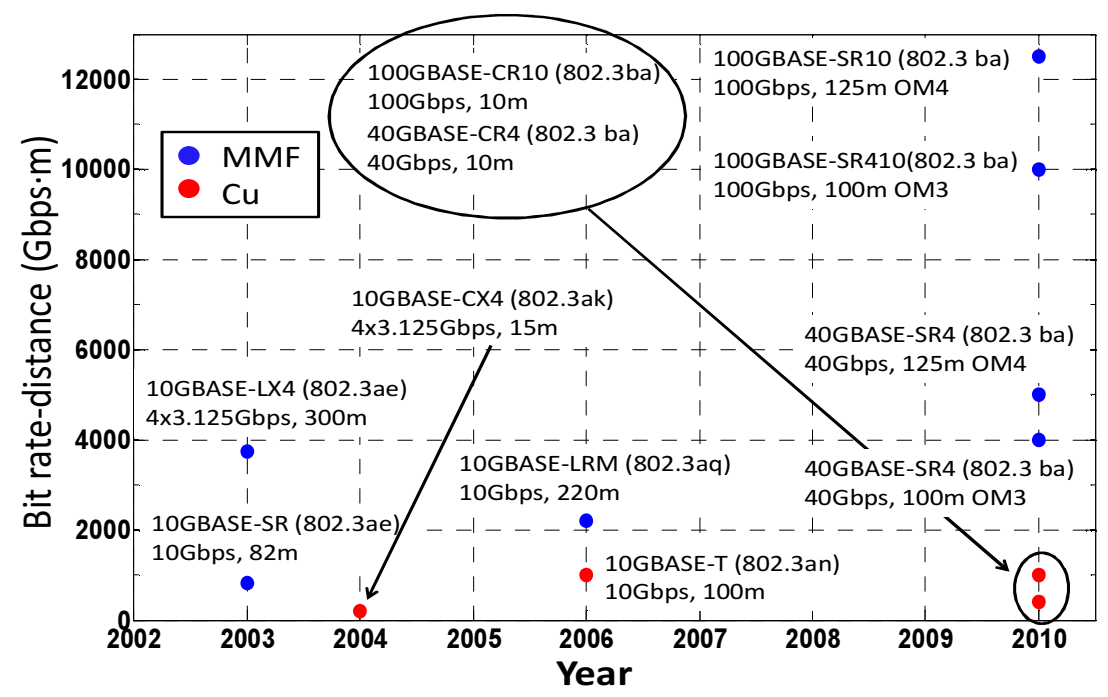

Figure 6. Gigabit Ethernet (10GbE) standards over MMF and copper links [44].

On the other hand, another important point in access networks communications is within the field of the wireless signal transmission (for both mobile and data communication), namely Wireless Local Area Networks (WLANs). Wireless technologies are developing fast but there is a need to link base stations/servers to the antenna by using fixed links together with the future exploitation of capacities well beyond present day standards (IEEE802.11a/b/g), which offer up to $54 \mathrm{Mbps}$ and operate at $2.4 \mathrm{GHz}$ and $5 \mathrm{GHz}$, as well as 3G mobile networks such as IMT2000/UMTS ${ }^{4}$, which offer up to 2Mbps and operate around 2GHz. Moreover, IEEE802.16, otherwise known as WiMAX, is another recent standard aiming to bridge the last mile through mobile and fixed wireless access to the end user at frequencies between 2-11GHz. In addition, WiMAX also aims to provide Fixed Wireless Access at bit-rate in the excess of $100 \mathrm{Mbps}$ and at higher frequencies between 10-66GHz. All these services use signals at the radio-frequency (RF) level that are analogue in nature, at least in the sense that they cannot be carried directly by digital baseband modulation. Optical cabling solutions can also offer the possibility for semi-transparent transport of these signals 
by using Radio-over-Fiber ( $\mathrm{RoF}$ ) technology. This RoF technology has been proposed as a solution for reducing overall system complexity by transferring complicated RF modem and signal processing functions from radio access points (RAPs) to a centralised control station (CS), thereby reducing system-wide installation and maintenance costs. Furthermore, although RoF in combination with multimode fibers can be deployed within homes and office buildings for baseband digital data transmission within the Ultra Wide Band (UWB), in general low carrier frequencies offer low bandwidth and the $6 \mathrm{GHz}$ UWB unlicensed low band is not available worldwide due to coexistence concerns [49]. These include radio and TV broadcasts, and systems for (vital) communication services such as airports, police and fire, amateur radio users and many others. In contrast, the $60 \mathrm{GHz}$-band, within millimetre wave, offers much greater opportunities as the resulting high radio propagation losses lead to numerous pico-cell sites and thus to numerous radio access points due to the limited cell coverage. These pico-cells are a natural way to increase capacity (i.e. to accommodate more users) and to enable better frequency spectrum utilisation. Therefore, for broadband wireless communication systems to offer the needed high capacity, it appears inevitable to increase the carrier frequencies even to the range of millimetre-wave and to reduce cell sizes [50]. Considering in-house wireless access networks, coaxial cable is very lossy at such frequencies and the bulk of the installed base of in-building fiber is silica-based MMF. Meanwhile PF GIPOF is also emerging as an attractive alternative, due to the aforementioned low cost potential and easier handling required in in-building networks. It is also mandatory to overcome the modal bandwidth limitation in multimode fibers to deliver modulated high frequency carriers to remote access points.

Following on this, it should be mentioned that PF GIPOFs have been demonstrated capable for transmission of tens of Gbps over distances of hundreds of meters. Some examples are reported in [51-53] in which more than 40Gbps over 100m of PF GIPOF are reported. An overview of some significant works over the years regarding GIPOF transmission can be seen in [54]. This is in contrast with all commercially available step-index POFs (SIPOFs) in which the bandwidth of transmission is limited to about $5 \mathrm{MHz} \cdot \mathrm{km} \mathrm{[6]} \mathrm{due} \mathrm{to} \mathrm{modal} \mathrm{disper-}$ sion. Therefore, even in the short-range communication scenario, the SIPOF is not able to cover the data rate of more than $100 \mathrm{Mbps}$ that would be necessary in many standards of the telecommunication area. Therefore, the SIPOF is mainly aimed at very short-range datarange transmission (less than $50 \mathrm{~m}$ ), image guiding and illumination.

\subsection{Multimode optical fiber expanded capabilities}

Although multimode fibers, both silica-based and polymer-based counterparts, are the best candidate for the convergence and achievement of a full service access network context, it has been previously addressed their main disadvantage concerning the limited bandwidth performance, limited by modal dispersion. For instance, for standard 62.5/125 $\mu \mathrm{m}$ silicabased MMFs, the minimum bandwidths are only specified to be $200 \mathrm{MHz} \cdot \mathrm{km}$ and $500 \mathrm{MHz}$. $\mathrm{km}$ (up to $800 \mathrm{MHz} \cdot \mathrm{km}$ ) in the $850 \mathrm{~nm}$ and $1300 \mathrm{~nm}$ transmission windows, respectively, under OverFilled Launch (OFL) condition ${ }^{5}$. Even though these specifications do satisfy the information rate of many classical short-range links, it is clear that a $2 \mathrm{~km}$-long campus 
backbone cannot be realized for operation at the speed of Gigabit Ethernet. This limited bandwidth hampers the desired integration of multiple broadband services into a common multimode fiber access or in-building/home network. Overcoming the bandwidth limitation of such fibers requires the development of techniques oriented to extend the capabilities of multimode fiber networks to attend the consumer's demand for multimedia services.

Headend station

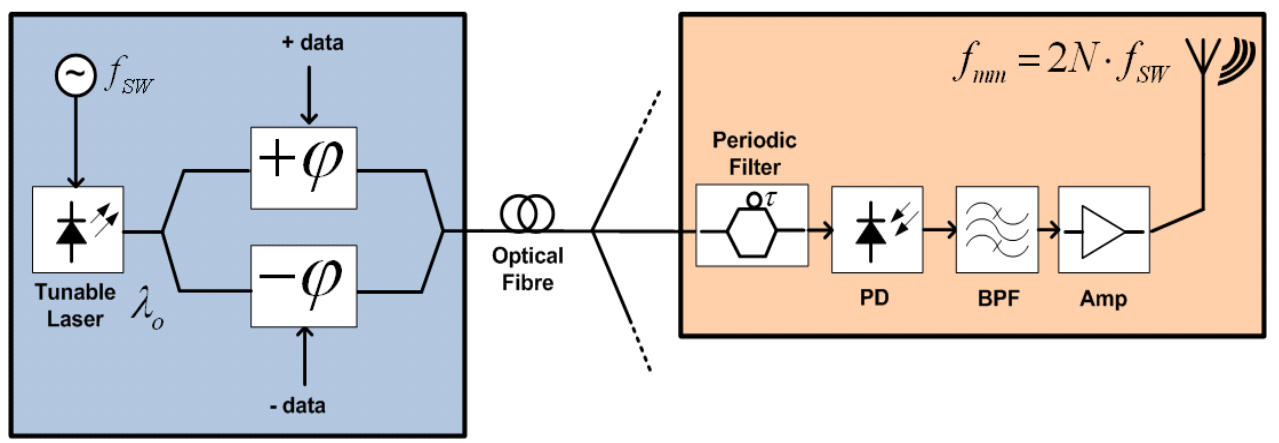

Figure 7. Feeding microwave data signals over a multimode network by OFM technique.

Novel techniques to expand the MMF capabilities and surmount this bandwidth bottleneck are continuously reported demonstrating that the frequency response of MMF does not diminish monotonically to zero after the baseband bandwidth, but tends to have repeated passbands beyond that [55]. In recent times, these high-order passbands and flat regions have been used in research to transmit independent streams of data (digital or analogue) complementary to the baseband bandwidth in order to exceed the aggregated transmission capacity of MMF [56] as well as to transport microwave and mm-wave radio carriers, commonly employed for creating high-capacity picocell wireless networks in RoF systems, as in [57]. Related to this latter technique, the Optical Frequency Multiplying (OFM) is a method by which a low-frequency RF signal is up-converted to a much higher microwave frequency through optical signal processing [58]. At the headend station, a wavelength-tunable optical source is used, of which the wavelength is periodically swept over a wavelength range with a sweep $f_{S W}$ while keeeping its output power constant. The data is then impressed on this wavelength-swept optical signal, see Fig. 7. After having passed through the optical fiber link, the signal impinges on a periodic optical multi-passband filter (e.g. optical comb or Fabry-Perot filter). In sweeping across $N$ transmission peaks of this filter (back and forth during one wavelength sweep cycle), light intensity burts arrive on the photodiode with a frequency $2 \cdot N \cdot f_{S W}$. Thus, the output signal of the photodiode contains a microwave frequency component at the above frequency and higher harmonics of which the strength depends on the bandpass characteristics of the periodic filter. Then, in order to select the desired har- 
monic, a bandapss filtering plus some amplification could be implemented. Note that only the optical sweep frequency is limited by the bandwidth of the optical fiber link, and that microwave carrier frequency can exceed this bandwidth by far due to the optical frequency multiplication mechanism. Extremely pure generated microwave signals have been demonstrated, notwithstanding a moderate laser spectral linewidth, due to the inherent phase noise cancellation in the OFM technique $[59,60]$.

On the other hand, subcarrier multiplexing (SCM) is a mature, simple, and cost effective approach for exploiting optical fiber bandwidth in analogue optical communication systems in general and RoF systems in particular. This technique was firstly addressed at the end of the 1990's in [61], which also takes advantage of the relative flat passband channels existing in the multimode fiber frequency response. Basically, in SCM, the RF signal (the subcarrier) is used to modulate an optical carrier at the transmitter's side. As a result, there is an optical spctrum consisting of the original optical carrier $f_{0}$ plus two side-tones located at $f_{0} \pm f_{S C}$, where $f_{S C}$ is the subcarrier frequency. If the subcarrier itlsef is modulated with data (either analogue or digital), then sidebands centered on $f_{0} \pm f_{S C}$ are produced. Finally, to multiplex multiple channels on to one optical carrier, multiple subcarriers are first combined and then used to modulate the optical carrier [62]. At the receiver's side the sucarriers are recovered through direct detection. One of the main advantages of SCM is that it supports broadband mixed mode data traffic with independent modulation format. Moreover, one subcarrier may carry digital data, while another may be modulated with an analogue signal, such as telephone traffic. However, the frequency ranges suitable for passband transmission vary from fiber to fiber as well as with the fiber length, the launching conditions or if the fiber is subjected to mechanical stress. Nevertheless, to overcome this limitation, an adaptative channel/allocation system would be necessary. Another drawback is that being SCM an analogue communication technique, it becomes more sensitive to noise effects and distortions due to non-linearities in the communications system.

It is worth noting that some other methods try to electrically improve this bandwidth performance using, for example, equalization techniques [63, 64]. In addition to, it is well known than an m-ary digital modulation scheme with $\mathrm{m}>2$ (multi-level coding) can enhance transmission capacity by overcoming the bandwidth limitations of a transmitter or a transmission medium and, therefore, multilevel modulation schemes that are used in radio-frequency communications have also been demonstrated in fiber-optic links [65]. Other attempts to overcome the bandwidth limit includes selective excitation of a limited number of modes, socalled Restricted Mode Launching (RML), in different ways: offset launch [66], conventional center launch [67] or even by means of a twin-spot technique [68]. Since the propagating modes are fewer under RML launch conditions, the difference in propagating times between the fastest and slowest modes is smaller, thus decreasing modal dispersion and increasing the corresponding bandwidth. In a similar way, Mode Group Diversity Multiplexing (MGDM) $[69,70]$ can be applied, in which the bandwidth increase is achieved by injecting a small light spot radially offset from the fiber core center thus limiting the number of modes excited within the fiber and, therefore, performing different simultaneous data transmission channels depending on the group of modes propagating. On the other hand, from the multimode fiber 
frequency response, the effect of having a wideband frequency-selective channel for data transmission can be overcome by using orthogonal frequency-division multiplexing (OFDM). In OFDM, the high-data-rate signal is error-correction encoded and then divided into many low-data-rate signals. By doing this, the wideband frequency-selective channel is separated into a series of many narrowband frequency-nonselective channels. OFDM technique has been applied to fiber-optic transmission [71] and shown to offer some protection against the frequency selectivity of a dispersive multimode fiber. Mode filtering techniques, either at the fiber input [72] or its output [73] have also been applied.

As cost is a key issue in local and residential networks, the use of Wavelength Division Multiplexing Passive Optical Network (WDM-PON) architectures for distribution of RoF signals has gained importance recently as WDM enables the efficient exploitation of the fiber network's bandwidth. This architecture acts as the starting point from the access node to the subscribing homes and buildings, constituting the all-optical fiber path. WDM-PON promises to combine both sharing feeder fibers while still providing dedicated point-to-point connectivity [74]. A basic scheme of the WDM-PON architecture can be seen in Fig. 1(b). In this case, optical microwave/mm-wave signals from multiple sources, which can be located in a Central Office (CO) or Optical Line Terminal (OLT) can be multiplexed and the composite signal is transported through an optical fiber and, finally, demultiplexed to address each Optical Network Terminal (ONT) or Remote Access Point (RAP), the latter for wireless applications. However, a challenging issue concerns the applications of these signals as the optical spectral width of a single mm-wave source may approach or exceed the WDM channel spacing.

Finally, it is worth mentioning that there is not the desire of making a competition between optical and wireless solutions, since wireless is and will always be present inside the building or home. In contrast, research and development are focusing on the coexistence of both technologies.

\section{Theoretical approach of multimode optical fibers}

\subsection{Introduction}

The restricted bandwidth of the multimode fiber has been one of the main causes that makes the specification and designing of the physical media dependent layer very difficult. Moreover, the potentials of MMFs to support broadband RF, microwave and millimetre wave transmission over short, intermediate and long distances to meet user requirements for higher data rates and to support emerging multimedia applications are yet to be fully known. To enable the design and utilization of MMFs with such enhanced speeds, the development of an accurate frequency response model to describe the signal propagation through multimode fibers is of prime importance. Through this multimode fiber modelling more likely performance limits can be established, thereby preventing eventual overdesign of systems and the resulting additional cost.

Since the mid-1970's, much work has been directed to the investigation of MMFs and their ability for high speed transmission. Different factors have clearly been identified to influ- 
ence the information-carrying capacity, namely the material dispersion (in combination with the spectrum of the exciting source) [26], the launching conditions [66] as well as the modedependent characteristics, i.e. delay [26], attenuation [75] and coupling coefficient [27]. Unfortunately, the achievements, so far accomplished, are not quite complete to enable precise frequency response and bandwidth prediction if an arbitrary operating condition is to be considered.

The most popular technique reported so far for the analysis of signal propagation through MMF fibers is that based on the coupled power-flow equations developed by Gloge [76] in the early 70's and later improved by Olshansky [27] and Marcuse [28], to account for the propagation and time spreading of digital pulses through MMFs. Most of the published models and subsequent work on the modelling of MMFs [29, 77-79] are based on this method in which the MMF power transfer function is solved by means of a numerical procedure like the Crank-Nicholson method, for instance [29]. However, other methods rely on solving the system of coupled equations adopting the matrix formalism [80].

The power-flow equations are adequate for the description of digital pulse propagation through MMFs but present several limitations either when considering the propagation of analogue signals or when a detailed knowledge of the baseband and RF transfer function is required since in these situations the effect of the signal phase is important. To overcome these limitations it is necessary to employ a method relying on the propagation of electric field signals rather than optical power signals. Unfortunately, there are very few of such descriptions available in the literature with the exception of the works reported in [54, 81, 82].

From literature, it is demonstrated that the frequency characteristics of multimode fibers should show significant high-frequency components, i.e. higher-frequency transmission lobes, resonances or passbands are expected in the fiber frequency response. And these higher-frequency transmission lobes would allow to transport information signals by modulating them on specific carrier frequencies, as an independent transmission channel each. These modulated carriers can be positioned in such a way that they will optimally fit into the higher-frequency transmission lobes of the multimode fiber link thus increasing the aggregated transmission capacity over MMFs. Furthermore, it has been stated that the contrast ratio between resonances reveals a dramatically reduction as the frequency increases thus providing potential for broadband transmission at even higher frequencies than those determined by the transmission lobes.

The position of these higher-frequency lobes depends on the fiber link length, and on the exact fiber characteristics, which may vary due to external circumstances such as induced stress by bending or environmental temperature variations. Any system that would take advantage of such high-frequency transmission lobes would have to adapt to those variations, e.g. monitoring the fiber link frequency response by injecting some weak pilot tones, and allocating the subcarriers accordingly would be a feasible solution. Anyway, this in turn is contingent on the availability of accurate models to describe the microwave radio signals propagation over multimode fibers. With such a predictive tool, notwithstanding its restricted bandwidth, a single multimode fiber network that may carry a multitude of broadband services using the higher-order transmission lobes would become more feasible. Thus, easy- 
to-install multimode fiber networks for access and in-building/home can be realised in which wirebound and wireless services were efficiently integrated.

\subsection{Mathematical framework}

In this section a closed-form analytic expression to compute the baseband and RF transfer function of a MMF link based on the electric field propagation method is briefly presented. By obtaining an accurate model it is possible to evaluate the conditions upon which broadband transmission is possible in RF regions far from baseband. For a deeper comprehension works reported in $[81,82]$ are recommended.

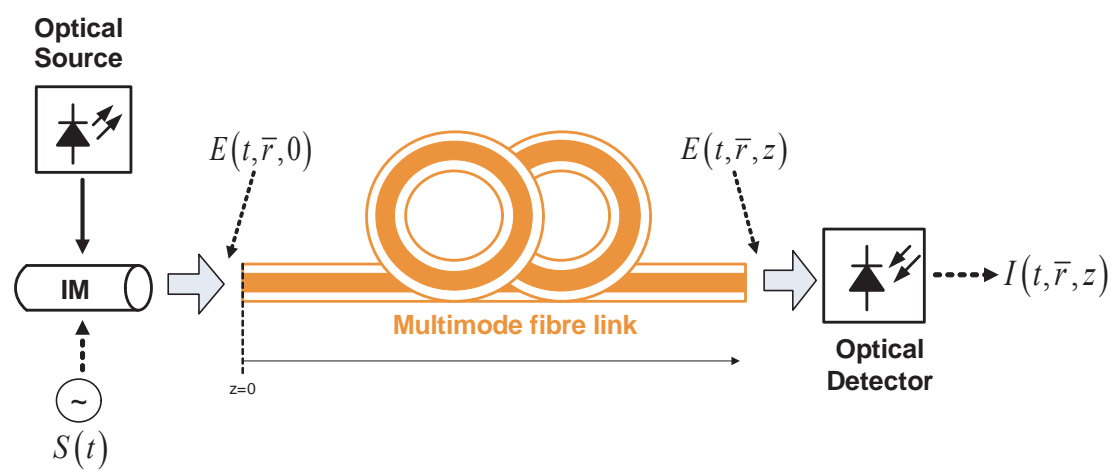

Figure 8. Scheme of a generic Multimode Optical Fiber link. IM: Intensity optical Modulator.

Fig. 8 shows a generic optical transmission system scheme which employs a multimode optical fiber as a transmission medium. $E(t, \bar{r}, z)$ represents the electric field at a point located at a distance $z$ from the fiber origin and at a point $\bar{r}$ of its cross section. $E(t, \bar{r}, 0)$ represents the electric field at the fiber origin and at a point $\bar{r}$ of its cross section and $S(t)$ is the modulation signal composed of a RF tone with modulation index $m_{0}$.

Thus the optical intensity at a point $z, I(t, \bar{r}, z)$, depends directly on the electric field $E(t, \bar{r}, z)$ at a point located at a distance $z$ from the fiber origin and at a point $\bar{r}$ of its cross section. Both the electric field and the optical intensity can be expressed, using the electric field propagation model and referred to the system described in Fig. 8, as [82]:

$$
\begin{aligned}
& E(t, \bar{r}, z)=\sum_{v=1}^{N} \sum_{\mu=1}^{N}\left[h_{\mu \nu}(t) * E_{v}(t, 0)\right] e_{v}(\bar{r}) \\
& I(t, \bar{r}, z) \propto\langle| E\left(t, \bar{r},\left.z\right|^{2}\right\rangle= \sum_{\mu=1}^{N} \sum_{v=1}^{N} \sum_{\mu^{\prime}=1}^{N} \sum_{v^{\prime}=1}^{N} e_{v}^{*}(\bar{r}) e_{v^{\prime}}(\bar{r}) . \\
& \cdot \int_{-\infty}^{\infty} \iint_{-\infty}^{\infty}\left\langle h_{\mu \nu}^{*}\left(t-t^{\prime}\right) h_{\mu^{\prime} v^{\prime}}\left(t-t^{\prime \prime}\right)\right\rangle\left\langle E_{\mu}^{*}\left(t^{\prime}, 0\right) E_{\mu^{\prime}}\left(t^{\prime \prime}, 0\right)\right\rangle d t^{\prime} d t^{\prime \prime}
\end{aligned}
$$


where $N$ is the number of guided modes, $h_{\mu \nu}(t)$ is the impulse response at $z$ caused by mode $v$ at the fiber origin over mode $\mu$ at $z$ and $e_{v}(r)$ is the modal spatial profile of mode $v$. It has been assumed that non linear effects are negligible.

Let $S(t)$ be the modulation signal composed of a RF tone with modulation index $m_{o}$ assuming a linear modulation scheme (valid for direct and external modulation), which incorporates the source chirp $\alpha_{C}$, and approximated by three terms of its Fourier series, following:

$$
\sqrt{S(t)}=\sqrt{S_{o}}\left\{1+\frac{m_{o}}{8}\left(1+j \alpha_{C}\right) e^{j \Omega t}+\frac{m_{o}}{8}\left(1+j \alpha_{C}\right) e^{-j \Omega t}\right\}
$$

where $S_{o}$ is proportional to the average optical power and $\Omega$ represents the frequency of the RF modulating signal. It has also been assumed an optical source which has a finite linewidth spectrum (temporal coherence) defined by a Gaussian time domain autocorrelation function.

Assuming a stationary temporal coherence of the source and assuming that the detector collects the light impinging on the detector area $A_{r}$, and produces an electrical current proportional to the optical power given by:

$$
P(t)=\int_{A_{r}} I(t, \bar{r}, z) d \bar{r}=\int_{-\infty}^{\infty} \int_{-\infty}^{\infty} \sqrt{S^{*}\left(t^{\prime}\right) S\left(t^{\prime \prime}\right)} \cdot Q\left(t-t^{\prime}, t-t^{\prime \prime}\right) d t^{\prime} d t^{\prime \prime}
$$

being

$$
Q\left(t^{\prime}, t^{\prime \prime}\right)=R\left(t^{\prime}, t^{\prime \prime}\right) Q_{O}\left(t^{\prime}, t^{\prime \prime}\right) \text { and } Q_{O}\left(t^{\prime}, t^{\prime \prime}\right)=\sum_{\mu=1}^{N} \sum_{v=1}^{N} \sum_{\mu^{\prime}=1}^{N} \sum_{v^{\prime}=1}^{N} C_{\mu \mu^{\prime}} \chi_{v v^{\prime}}\left\langle h_{\mu v}^{*}\left(t^{\prime}\right) h_{\mu^{\prime} v^{\prime}}\left(t^{\prime \prime}\right)\right\rangle
$$

From the above equations:

- The term $Q\left(t^{\prime}, t^{\prime \prime}\right)$ is referred to the influence of the source/fiber/detector system.

- The term $Q_{O}\left(t^{\prime}, t^{\prime \prime}\right)$ depends on the fiber and the power coupling from to the source to the fiber and from the fiber to the detector.

- The spatial coherence of the source related to the fiber modes is provided by $C_{\mu \mu}$ '

- $\chi_{v v^{\prime}}$ is defined as $\chi_{v v^{\prime}}=\int_{A_{r}} e_{v}^{*}(\bar{r}) e_{v}{ }^{\prime}(\bar{r}) d \bar{r}$. In the special case where the detector collects all the incident light $\chi_{v v^{\prime}}=\delta_{v v^{\prime}}$. 
- The term $\left\langle h_{\mu \nu}^{*}\left(t-t^{\prime}\right) h_{\mu^{\prime} v^{\prime}}{ }^{\prime}\left(t-t^{\prime \prime}\right)\right\rangle$ is referred to the fiber dispersion and to the mode coupling.

This last term, relative to the propagation along the fiber, is composed of two parts, one describing the independent propagation of modes $h_{\mu \nu}^{*}\left(t-t^{\prime}\right)$ and a second one describing the power coupling between modes $h_{\mu^{\prime} v^{\prime}}\left(t-t^{\prime \prime}\right)$. For analysing this term, it is required to consider the $\mathrm{N}$ coupled mode propagation equations (field amplitudes) in the frequency domain which refer to an $\mathrm{N}$-mode multimode fiber. A detailed study of this analysis can be found in $[54,82]$.

Although Eq. (14) reveals a nonlinear relationship between the output and the input electrical signals being not possible to define a transfer function, under several conditions linearization is possible yielding to a linear system with impulse response $Q(t)$. This linear response is given by [81]:

$$
P(t)=\int_{-\infty}^{\infty} S\left(t^{\prime}\right) Q\left(t-t^{\prime}, t-t^{\prime}\right) d t^{\prime}
$$

The impulse response terms of the fiber can then be found by inverse Fourier transforming the above matrix elements. Upon substitution in Eq. (16) it is found that $P(t)$ is composed of two terms $P(t)=P^{U}(t)+P^{C}(t)$ being $P^{U}(t)$ the optical power in absence of mode coupling and $P^{C}(t)$ the contribution of modal coupling. Moreover, both the coupled and uncoupled parts can be divided into a linear and a non-linear term, respectively. These non-linear terms will contribute to the harmonic distortion and intermodulation effects. Grouping the linear contributions of the uncoupled and the coupled parts, and comparing the power of the lineal part of the total power received (sum of contributions from the coupled and uncoupled parts) with the power of one of the sidebands of the electric modulating signal, it is possible to obtain the final overall RF transfer function, yielding Eq. (17). For a detailed description of the evaluation of both terms, see the works reported in [54, 82].

$$
H(\Omega)=\sqrt{1+\alpha_{C}^{2}} \cdot e^{-\frac{1}{2}\left(\frac{\beta_{0}^{2} \Omega z}{\sigma_{c}}\right)^{2}} \cdot \cos \left(\frac{\beta_{o}^{2} \Omega^{2} z}{2}+\arctan \left(\alpha_{C}\right)\right) \cdot \sum_{m=1}^{M} 2 m\left(C_{m m} \chi_{m m}+G_{m m}\right) e^{-2 \alpha_{m} z} e^{-j \Omega \tau_{m} z}
$$

The expression of Eq. (17) provides a description of the main factors affecting the RF frequency response of a multimode fiber link and can be divided as the product of three terms of factors. From the left to the right, the first term is a low-pass frequency response which depends on the first order chromatic dispersion parameter $\beta_{o}^{2}$ which is assumed to be equal for all the modes guided by the fiber, and the parameter $\sigma_{C}$ which is the source coherence time directly related to the source linewidth. The second term is related to the Carrier Sup- 
pression Effect (CSE) due to the phase offset between the upper and lower modulation sidebands, as the optical signal travels along a dispersive waveguide, i.e. optical fiber. When the value of this relative phase offset is 180 degrees, a fading of the tone takes place. Finally, the third term represents a microwave photonic transversal filtering effect [83], in which each sample corresponds to a different mode group $m$ carried by the fiber. Coefficients $C_{m m}, \chi_{m m}$ and $G_{m m}$ stand for the light injection efficiency, the mode spatial profile impinging the detector area and the mode coupling coefficient, respectively. This last term involves that the periodic frequency response of transversal filters could permit broadband RF, microwave and mm-wave transmissions far from baseband thus achieving a transmission capacity increase in such fiber links. Parameters $\alpha_{m m}$ and $\tau_{m m}$ represent the differential mode attenuation (DMA) effect and the delay time of the guided modes per unit length, respectively.

\section{Analysis and results on silica-based multimode optical fibers}

The MMF transfer function presented in Eq. (17) provides a description of the main factors affecting the RF frequency response of a multimode fiber link, including the temporal and spatial source coherence, the source chirp, chromatic and modal dispersion, mode coupling (MC), signal coupling to modes at the input of the fiber, coupling between the output signal from the fiber and the detector area, and the differential mode attenuation (DMA). Theoretical simulations and experimental results are studied with regards to several parameters in order to determine the optimal conditions for a higher transmission bandwidth in baseband and to investigate the potencials for broadband Radio-over-Fiber (RoF) systems in regions far from baseband using multimode fiber.

For the simulation results in this section it has been considered a $62.5 / 125 \mu \mathrm{m}$ core/cladding diameter graded-index multimode fiber (GI-MMF) with a typically $\mathrm{SiO}_{2}$ core doped with 6.3 mol- $\% \mathrm{GeO}_{2}$ and $\mathrm{SiO}_{2}$ cladding, and intrinsic attenuation of $0.55 \mathrm{~dB} / \mathrm{km}$. This typical doping value has been provided by the manufacturer. The refractive indices were approximated using a three-term Sellmeier function for $1300 \mathrm{~nm}$ and $1550 \mathrm{~nm}$ wavelengths. Sellmeier coefficients were provided by the manfacturer. Core and cladding refractive indices as a function of wavelength, from the Sellemier equation, Eq. (3), are illustrated in Fig. 9. A comparison of the core refractive index for a different core doped multimode fiber consisting of $7.5 \mathrm{~mol}-\%$ $\mathrm{GeO}_{2}$ is given. The parameters relative to the differential mode attenuation were fitted to $\rho=9$ and $\eta=7.35$. Coefficient $G_{m m}$ was obtained assuming a random coupling process defined by a Gaussian autocorrelation function [28] with a rms deviation of $\sigma=0.0009$ and a correlation length of $\varsigma=115 \cdot a$, being $a$ the fiber core radius. The rms linewidth of the source was set to $10 \mathrm{MHz}$ and its chirp parameter to zero. A refractive index profile of $\alpha=2$ was considered. Overfilled launching condition (OFL) was also assumed so that the light injection coefficient was set to $C_{m m}=1 / M$, being $M$ the total number of mode groups.

Fig. 10 illustrates the frequency response of a 3km-long GI-MMF link in absence and presence of DMA and mode coupling effects. An optical source operating at 1300nm and with 


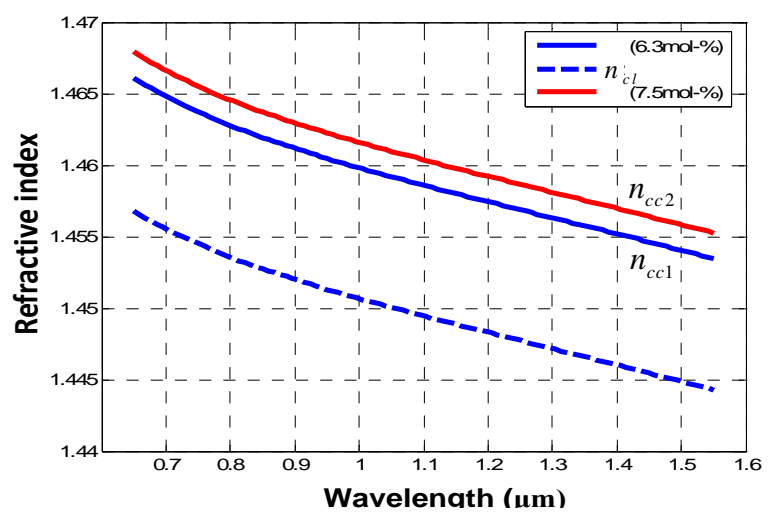

Figure 9. MMF core $\left(n_{c c i}\right)$ and cladding $\left(n_{c l}\right)$ refractive index for different dopant concentration.

$10 \mathrm{MHz}$ of linewidth has been considered. The filtering effect caused by the DMA is decreased when considering the presence of the mode coupling phenomenon. Moreover, the RF baseband bandwidth is increased by mode coupling while DMA has little effect on the bandwidth itself. Anyway, not considering mode coupling effects, Fig. 10 illustrates the classical conflict relationship between dispersion and loss in MMFs in general. As a matter of fact, the large DMA of high-order modes necessarily causes a large power penalty during light propagation, but at the same time it yields a bandwidth enhancement as a result of the mode stripping effect. Finally if mode coupling effects are considered, there is no deviation on the resonance central frequencies no matter the fiber DMA whilst DMA has a significant effect when mode coupling is considered to be negligible. $\left.f_{o}\right|_{n}$ refers to the possible transmission channels far from baseband that could be employed.

(a)

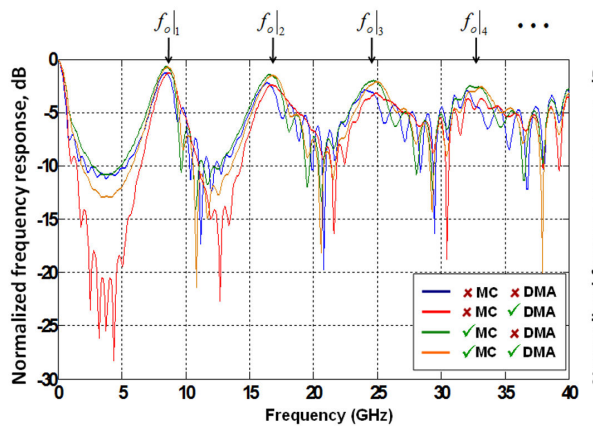

(b)

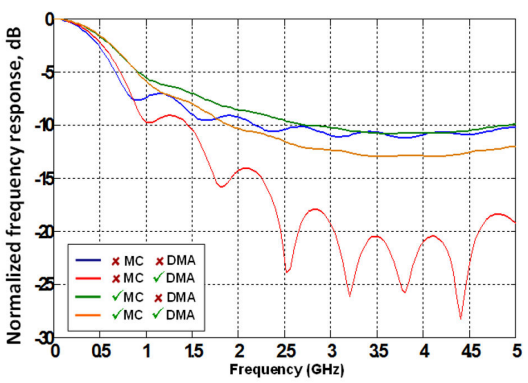

Figure 10. (a) Frequency responses up to $40 \mathrm{GHz}$ for a $62.5 / 125 \mu \mathrm{m}$ GIMMF showing the effect of mode coupling and DMA. L=3km. (b) Zoom up to $5 \mathrm{GHz}$. 
The influence of the optical fiber properties over its frequency response is of great importance. Parameters such as the core radius, the graded-index exponent, length and the core refractive index count for this matter. Nevertheless, the most critical parameter that define the behaviour and performance of a graded-index optical fiber type is its refractive index profile $\alpha$. It should be outlined that this index profile may slightly vary with wavelength, always due to the eventually nonlinear Sellmeier coefficients. As a consequence of this, a profile conceived to be optimal (in terms of bandwidth, for example) at a given wavelength may will be far from optimal at another wavelength. This fact was also addressed in Section 2.2. The $\alpha$-dispersion is imposed by the dopant and its concentration, so this impairment is not easy to overcome. Furthermore, these latter parameters can also be affected by temperature impairments, as recently reported in [84]. Frequency responses are displayed in Fig. 11 (a) for a $2 \mathrm{~km}$-long GI-MMF link showing the influence of $1 \%$ fiber refractive index profile deviations on the RF transfer function. The rest of parameters for the simulations take the same value as aforementioned. Significant displacements of the high-order resonances over the frequency spectrum are noticed. From simulation conditions, attending to Fig. 11(a), an increase of $\alpha^{\prime}=\alpha+0.04$ produces a change of the first-order resonance central frequency of $3.2 \mathrm{GHz}$. It is also noticeable that the $3-\mathrm{dB}$ passband bandwidth of the high-order resonances is also highly influenced. Both facts could cause a serious MMF link fault if multiple- $\mathrm{GHz}$ carriers are intended to be transmitted through this physical medium when performing a RoMMF system.

(a)

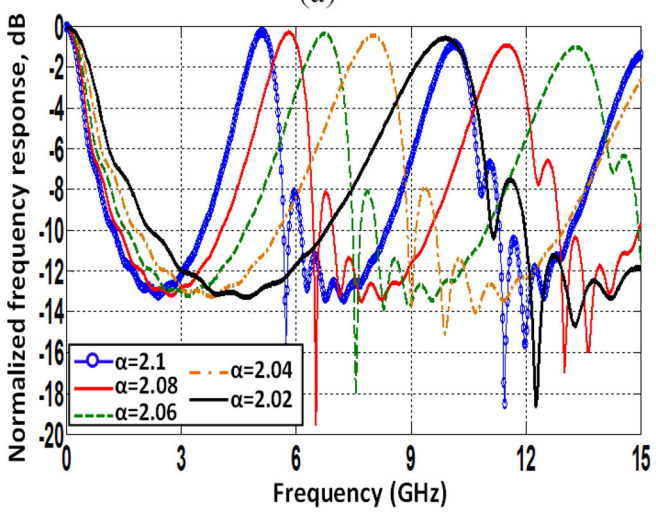

(b)

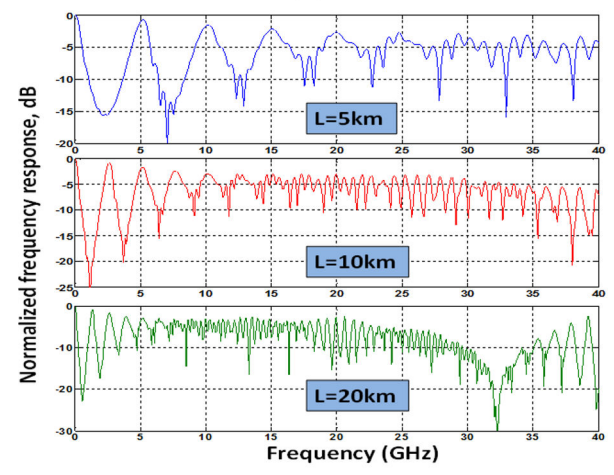

Figure 11. (a) Influence of the refractive index profile on the GI-MMF frequency response for a $2 \mathrm{~km}$-long link. (b) GIMMF frequency response for different link lengths, covering access reach.

The MMF frequency response dependence on the link length is shown in Fig. 11(b), covering typical access network distances. High-order resonances far from baseband are slightly displaced over the frequency spectrum with changes in attenuation depending on the case. In addition, transmission regions can be easily identified as well as the effect of the carrier sup- 
pression (CSE) due to the presence of intermediate notches, as seen in the case of $\mathrm{L}=20 \mathrm{~km}$. This effect can not be overlooked but could be avoided using single sideband modulation

Finally, the following figures illustrate both the influence of the optical source linewidth characteristic as well as the launching condition with regards to the GI-MMF frequency response. The influence of other optical source characterisitics such as the source chirp and the operating wavelength can be seen in [54]. It should be noted that wavelength emission provided by the optical source links with different optical fiber properties to be considered. Parameters such as the core and cladding refractive indices, the material dispersion, the propagation constant, the intrinsic attenuation and the number of propagated modes strongly depend on the optical wavelength launched into the fiber, being not an easy task to determine a real comparison about the influence of this parameter on the frequency response.

Fig. 12(a) illustrates the GI-MMF frequency response of a $3 \mathrm{~km}$-long link for three different optical sources operating at $1300 \mathrm{~nm}$. The rest of parameters take the same value as those previously indicated. The response for the DFB laser (with a Full Width Half Maximum FWHM- of $10 \mathrm{MHz}$ ) behaves relatively flat at high frequencies. The frequency response employing a FP laser with 5.5nm linewidth however suffers from a low-pass effect, determined by a $40 \mathrm{~dB}$ fall at $40 \mathrm{GHz}$. In the case of using a broadband light source, such a Light Emitting Diode (LED) with 30nm of source linewidth, the response falls dramatically after a few $\mathrm{GHz}$ and no high-order resonances are observed. On the other hand, the influence of the launching condition on the frequency response can be seen in Fig. 12(b). A GI-MMF link of $1 \mathrm{~km}$ and an input power spectral density conforming a Gaussian lineshape from a DFB optical source with $10 \mathrm{MHz}$ FWHM have been considered. From the frequency response it is noticeable the dramatic enhancement of the baseband bandwidth as well as the achievement of a flat response in all the $20 \mathrm{GHz}$-spectrum considered.

(a)

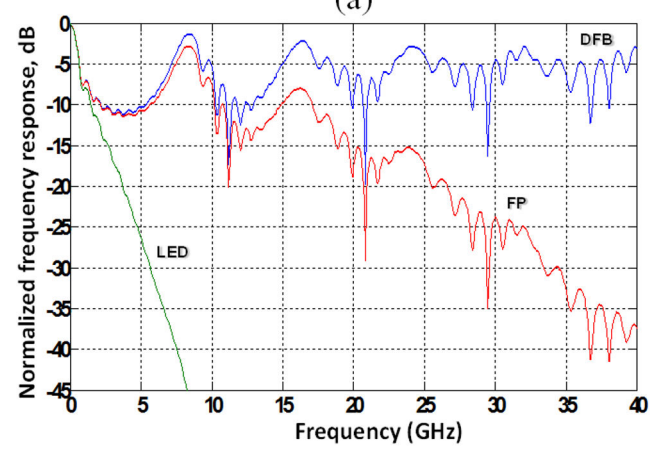

(b)

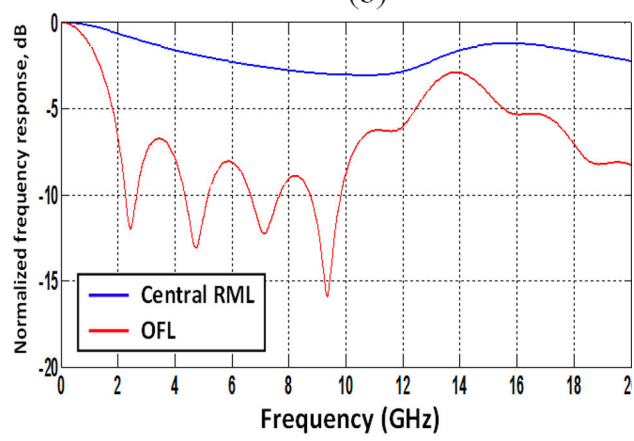

Figure 12. (a) Influence of the optical source temporal coherence on the frequency response of a $3 \mathrm{~km}$-long GI-MMF link. (b) Influence of the light injection on the frequency response of a $1 \mathrm{~km}$-long GI-MMF link.

By evaluating the latter results, it is observed that exploiting the possibility of transmitting broadband signals at high frequencies is contingent on the use of both narrow-linewidth op- 
tical sources and selective mode-launching schemes. These requirements were also confirmed in the work reported in [82].

Some measurement examples of the silica-based MMF transfer function are presented highlighting the conditions upon broadband MMF transmission in regions far from baseband can be featured thus validating the theoretical model described and proposed in [81]. The setup schematic for the experimental measurements is shown in Fig. 13.

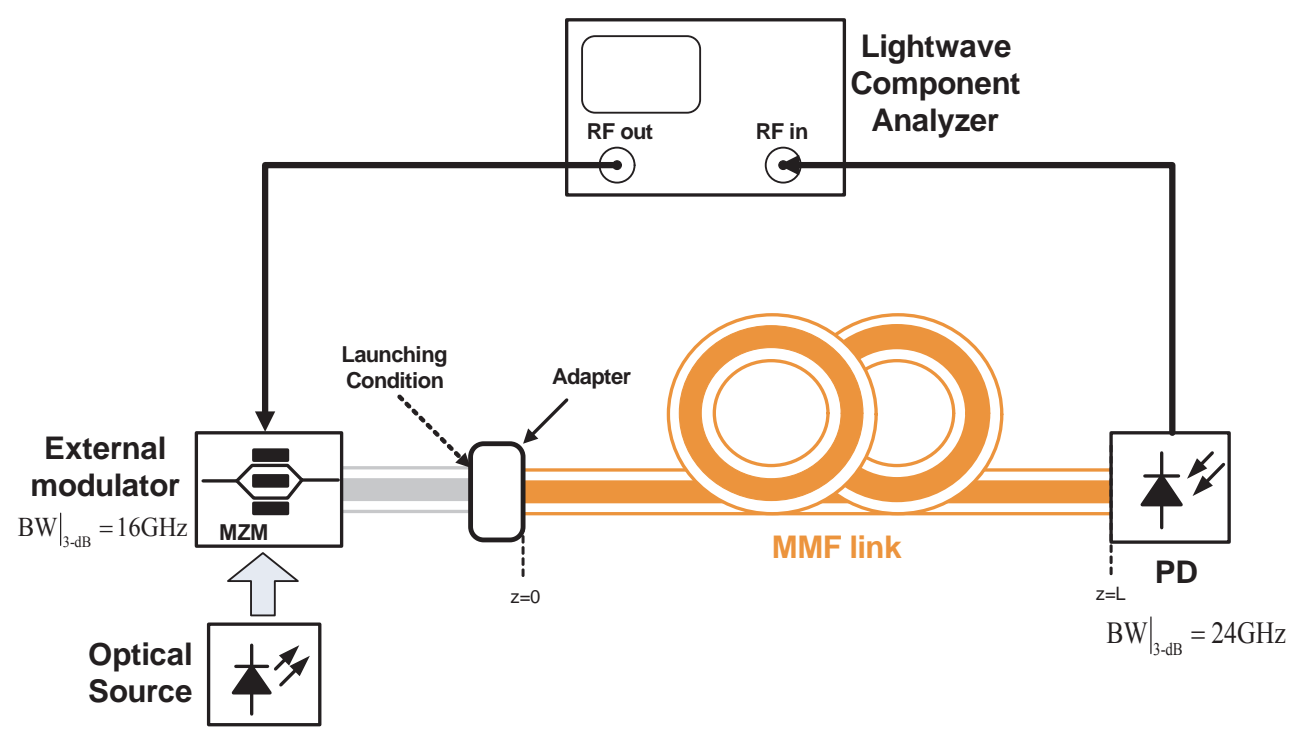

Figure 13. Block diagram of the experimental setup for the silica-based GI-MMF frequency response measurement up to $20 \mathrm{GHz}$.

A Lightwave Component Analyzer (LCA, Agilent 8703B, 50MHz-20GHz) has been used to measure the frequency response, using a $100 \mathrm{~Hz}$ internal filter. In all cases the laser was externally intensity modulated with an RF sinusoidal signal up to $20 \mathrm{GHz}$ of modulation bandwidth, by means of an electro-optic (E/O) Mach-Zehnder modulator (model JDSU AM-130@1300nm and JDSU AM-155@1550nm). At the receiver, the frequency response is detected by using a high-speed PIN photodiode, model DSC30S, from Discovery Semiconductors. It should be mentioned that the experimental results of the silica-based GI-MMF link shown in this section have been calibrated with regards to both the E/O intensity modulator and the photodetector electrical responses, being therefore solely attributed to the MMF fiber. It should be also noted that the ripples observed are caused by reflections in the optical system and are not features of the fiber response, although FC/APC connectors are used to minimize this effect. To perform different launching conditions, the optical output of the E/O modulator was passed through a $62.5 / 125 \mu \mathrm{m}$ silica-based MMF fiber patch cord plus a mode scrambler before being launched to the MMF link. This optical launching 
scheme provides an OFL condition for light injection. On the other hand, selective central mode launching was achieved by injecting light to the system via a SMF patchcord.

Fig. 14(a) shows the measured frequency response for a $3 \mathrm{~km}$ silica-based GI-MMF link. As it was expected from the theory, while the response for the DFB laser (@1550nm) behaves relatively flat at high frequencies, with maximum variations of approximately $\pm 0.8 \mathrm{~dB}$ with regards to a mean level of approximately $2.5 \mathrm{~dB}$ below the low frequency regime, the response relative to the FP laser (@1310nm) suffers from a low pass effect characterized by a $15 \mathrm{~dB}$ fall at $20 \mathrm{GHz}$. In the case of the Broadband Light Source (BLS), the response falls dramatically after a few GHz. Therefore, as previously stated, exploiting the possibility of transmitting broadband RF signals at high frequencies is contingent on the use of narrow-linewidth sources. This latter performance stands regardless the operating wavelength from the optical source.

(a)

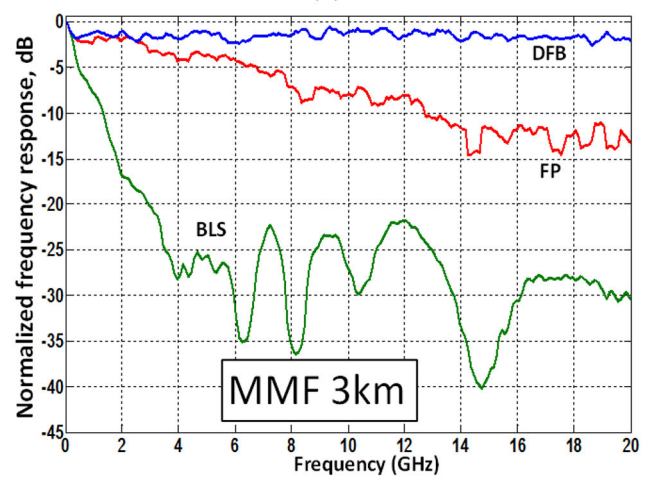

(b)

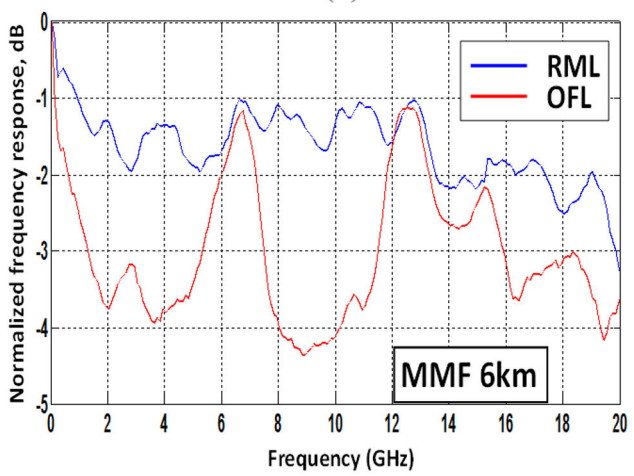

Figure 14. (a) Measured influence of the optical source linewidth on the silica-based GI-MMF frequency response. (b) Measured influence of the launching condition on the silica-based GI-MMF frequency response.

Additionally, two launching conditions, RML and OFL, were also applied to the fiber link. Results are shown in Fig. 14(b), and were performed by using a DFB laser operating at with FWHM of $100 \mathrm{kHz}$. As expected, for the RML condition, in which a limited number of modes is excited, the typical transversal filtering effect of the MMF is significantly reduced, thus achieving an increased flat response over a broader frequency range spectrum. It should be noted that the distance values comprising Fig. 14 are representative of currently deployed moderate-length fiber links.

Finally, the above figures show a comparison between the curves predicted by the theoretical model and the experimental results showing good agreement between them. A FP source operating at $1300 \mathrm{~nm}$ with $\Delta \lambda=1.8 \mathrm{~nm}$ of source linewidth has been employed in measurements reported in Fig. 15(a). An OFL excitation at the fiber input end has been applied. Theoretical curves have been obtained considering a silica-based MMF with a $\mathrm{SiO}_{2}$ core doped with $6.3 \mathrm{~mol}-\% \mathrm{GeO}_{2}$ and a $\mathrm{SiO}_{2}$ cladding, with a refractive index profile of 
(a)

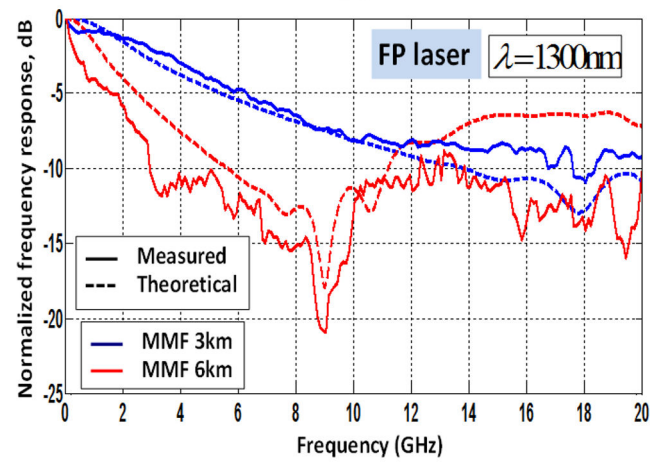

(b)

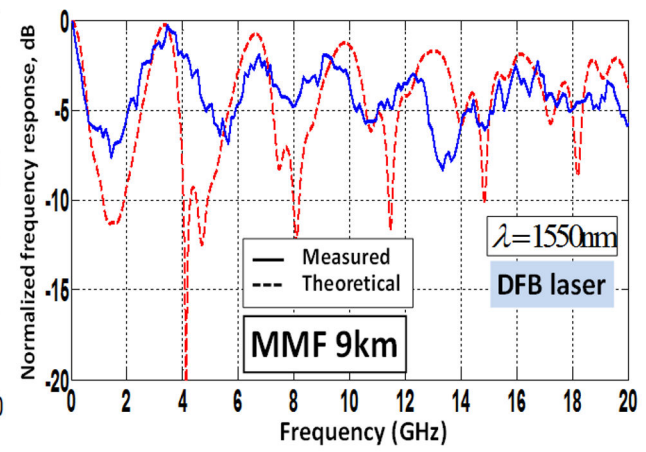

Figure 15. (a) Theoretical and measured frequency response of a $3 \mathrm{~km}$ - and $6 \mathrm{~km}$-long silica based GI-MMF link with a FP laser source operating at $1300 \mathrm{~nm}$. (b) Theoretical and measured frequency response of a $9 \mathrm{~km}$-long silica-based GIMMF link with a DFB laser source operating at 1550nm [84].

$\alpha=1.921$ and an intrinsic attenuation coefficient of $\alpha_{o}=0.59 \mathrm{~dB} / \mathrm{kmat} 1300 \mathrm{~nm}$. The latter was measured employing Optical Time-Domain Reflectometer (OTDR) techniques. Core and cladding refractive indices have been calculated using a three-term Sellmeier function. It has also been assumed a free chirp source. Differential Mode Attenuation (DMA) effects have been considered by setting $\rho=8.7 ; \eta=7.35$. Additionally, a random coupling process defined by a Gaussian autocorrelation function has been defined for the mode coupling with a correlation length of $\varsigma=0.0036 \mathrm{~m}$ and rms deviations of $\sigma=0.0012 @ 3 \mathrm{~km}$ and $\sigma=0.0017 @ 6 \mathrm{~km}$. Fig. 15(a) also addresses the high-order resonances (passband) suppression effect as the source linewidth increases.. This is due to the fact that in this latter case the low pass term in Eq. (17) begins to dominate over the other two. In constrast, in Fig. 15(b), a DFB laser source with $100 \mathrm{kHz}$ of linewidth and operating at $1550 \mathrm{~nm}$ has been employed. An intrinsic fiber attenuation coefficient of $\alpha_{o}=0.31 \mathrm{~dB} / \mathrm{km}$ at $1550 \mathrm{~nm}$ was measured and a rms deviation of $\sigma=0.0022 @ 9 \mathrm{~km}$ was considered for a link length of $9 \mathrm{~km}$. The rest of parameters take the same values as aforementioned. Several passband channels suitable for multiple-GHz carrier transmission over the frequency spectrum are observed as well as a relatively flat region over 17GHz. However, a significant discrepancy can be observed in the resonances excursions, being the measured ones not so pronounced compare to what the model predicts, i.e. the measured filtering effect is decreased compare to what it is expected. Many reasons can be attributed for this behaviour but mainly due to both the DMA and mode coupling modelling approaches considered.

Finally, although the 3-dB bandwidth of the baseband frequency response has not been paid much attention in this analysis, it is commonly agreed that the measurement uncertainty in characterizing this parameter is quite large and a standard deviation on the order of $10 \%-20 \%$ or more is not uncommon. This performance depends on the care of a particular lab in setting up the launch conditions and acquiring the data. This was verified in 1997 with 
an informal industry wide round robin [85]. Furthermore, it was, in fact, because of this that the industry standardized the overfilled launch (OFL) condition during the late 1990s [86].

\section{Analysis and results on graded-index polymer optical fibers}

This section, comprising Graded-Index Polymer Optical Fibers (GIPOFs) will follow the same scheme as previous section. Furthermore, this section proves that the same principles are essentially valid for silica-based MMFs and GIPOFs in order to extend their capabilities beyond the RF baseband bandwidth.

For the simulation results in this section it has been considered a 120/490 $\mu$ m core/cladding diameter graded-index polymer optical fiber (PF GIPOF) with intrinsic attenuation of $60 \mathrm{~dB} / \mathrm{km}$ at $1300 \mathrm{~nm}$ and $150 \mathrm{~dB} / \mathrm{km}$ at $1550 \mathrm{~nm}$. The refractive indices for the fiber core and fiber cladding were calculated using a three-term Sellmeier. These coefficients were provided by the manfacturer. Core and cladding refractive indices as a function of wavelength, from the Sellemier equation, Eq. (3), are illustrated in Fig. 16. The parameters relative to the differential mode attenuation were fitted to $\rho=11$ and $\eta=12.2$. Coefficient $G_{m m}$ was obtained assuming a random coupling process defined by a Gaussian autocorrelation function with a rms deviation of $\sigma=0.0005$ and a correlation length of $\varsigma=1.6 \times 10^{4} \cdot a$, being $a$ the fiber core radius. This latter value of the correlation length provides similar mode coupling strengths than that of reported in other works for PF GIPOF fibers such as in [35, 44]. The rms linewidth of the optical source was set to $5 \mathrm{~nm}$ and its chirp parameter to zero. A refractive index profile of $\alpha=2$ was considered, unless specified. Overfilled launching condition (OFL) was also assumed so that the light injection coefficient was set to $C_{m m}=1 / M$, being $M$ the total number of mode groups.

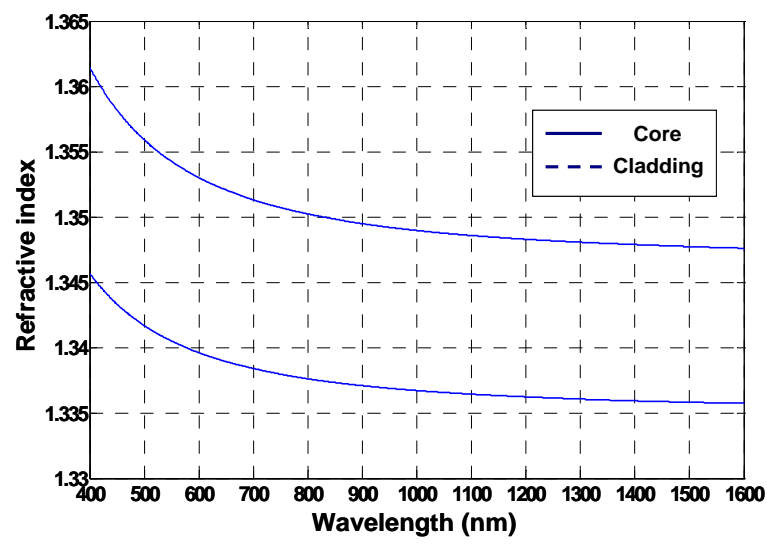

Figure 16. PF GIPOF core and cladding refractive index as a function of wavelength. 
It is worth mentioning that with PF GIPOFs, a thermally determined alteration in the dopant material can come about, leading to changes in the refractive index, although new materials have just recently become available and behave with admirable stability in this issue [87]. This dopant concentration during the manufacturing process is also directly related to the fiber refractive index profile.

Fig. 17(a) depicts the frequency response of a 200m-long PF GIPOF link operating at 1300nm in absence and presence of DMA and mode coupling effects. The theoretical curve for a $200 \mathrm{~m}-$ long PMMA GIPOF in presence of both DMA and mode coupling is also given for comparison. As expected, much greater baseband bandwidths are obtained by using fluorine dopants in the core instead of classical PMMA-based composites. The results indicate that the presence of both effects is favorable for improving the frequency response of the GIPOF. It can be observed a more than a three-fold RF baseband bandwidth enhancement caused if only DMA effect is considered. This result shows that the DMA is a determining factor for accurate assessment of the baseband in GIPOFs. No high-order resonances are shown due to both the high fiber attenuation and the OFL launching condition considered. As in the case of silicabased MMFs, the influence of the optical fiber properties over its frequency response is of great importance. Parameters such as the core radius, the graded-index exponent, length and the core refractive index count for this matter. Similar mechanisms as those stated for silicabased GI-MMFs rule for PF GIPOFs concerning these parameters. This fact is illustrated in Fig. 17(b), in which PF GIPOF frequency responses are displayed for a 200m-long link showing the influence of $5 \%$ fiber refractive index profile deviations on the RF transfer function. The rest of parameters for the simulations take the same value as aforementioned. Similarly to silicabased counterparts, significant displacements of the high-order resonances over the frequency spectrum are noticed. However, it is worth pointing out that PF GIPOFs are less sensitive to $\alpha$-tolarences compared to that of silica counterparts.

(a)

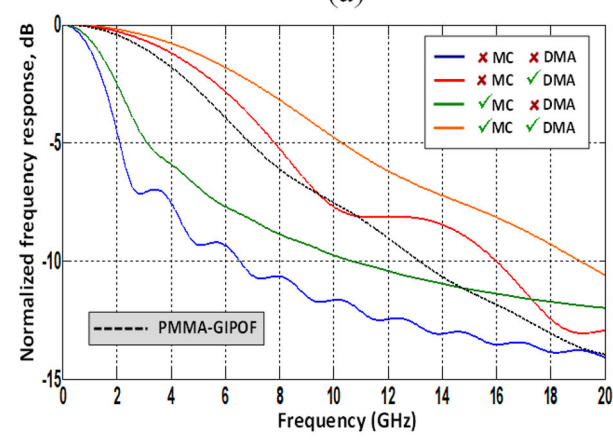

(b)

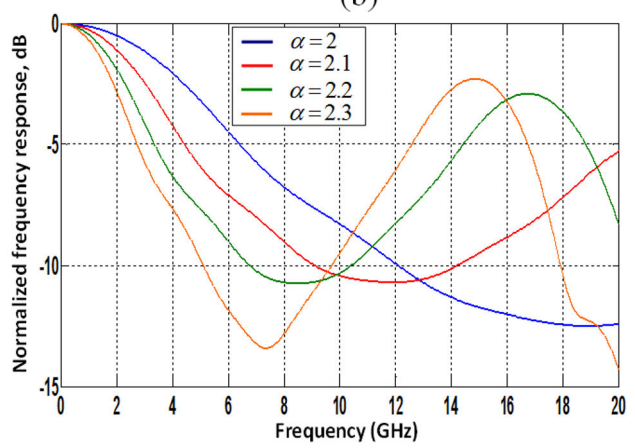

Figure 17. (a) Frequency responses up to $20 \mathrm{GHz}$ for a $200 \mathrm{~m}$-long PF GIPOF link showing the effect of mode coupling and DMA. Similar PMMA-based link is also illustrated for comparison. (b) Influence of the refractive index profile on the PF GIPOF frequency response for a $200 \mathrm{~m}$-long link. 
(a)

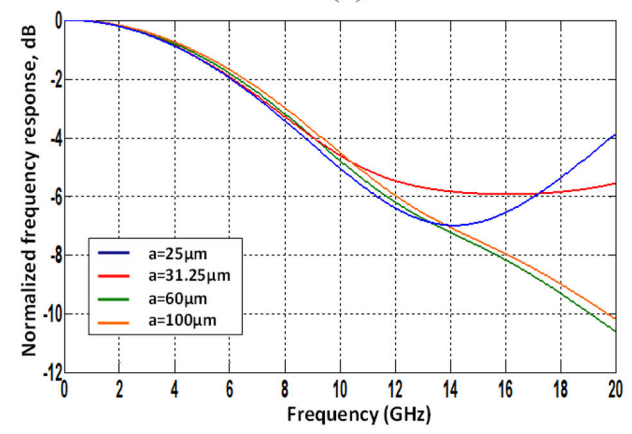

(b)

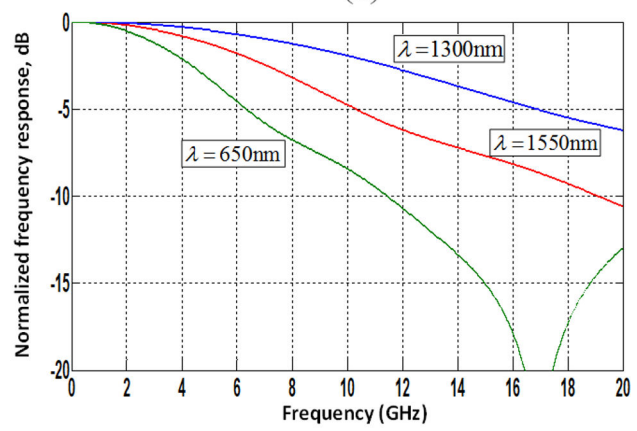

Figure 18. (a) Influence of the core radius on the PF GIPOF frequency response at OFL condition. (b) Influence of the operating wavelength on the PF GIPOF frequency response.

On the other hand, Fig. 18(a) illustrates the frequency response of present commercially available PF GIPOFs with different core radius. Identical simulation parameters have been considered. From the theoretical curves, similar RF baseband bandwidths at OFL condition are obtained, independent from the core radius considered, although high-order resonances start to notice as core radius decreases. However, this fact turns to be different if RML launching is applied. Simulations under this light injection condition predict that lower fiber core radius results in a RF baseband bandwidth enhancement. This result is quite in agreement with the fact that the bandwidth reduction is to be connected with the larger number of excited modes, directly related to the fiber core radius. Nevertheless, this dependence is strongly reduced as nearer OFL is reached. Moreover, the frequency response dependence on the operating wavelength is shown in Fig. 18(b). As expected, due to the high chromatic dispersion of PF GIPOFs at $650 \mathrm{~nm}$, see Fig. 3(a), RF baseband bandwidth at this wavelength falls dramatically after few GHz. On the other hand, baseband bandwidths achievable at $1300 \mathrm{~nm}$ are greater than those obtained at 1550nm despite the similar PF GIPOF material dispersion (even slight smaller at 1550nm) and despite the use of a relatively narrow-linewidth optical source. Thus, bandwidth must mostly be limited by modal dispersion. The reason for this bandwidth difference is supported by the fact that DMA effects are supposed to be stronger at $1300 \mathrm{~nm}$ than that of 1550nm, leading to a RF baseband bandwidth enhancement.

Finally, the following figures illustrate both the influence of the optical source linewidth characteristic as well as the launching condition with regards to the PF GIPOF frequency response. The influence of other optical source characterisitics such as the source chirp and the operating wavelength can be seen in [54]. Fig. 19(a) illustrates the PF GIPOF frequency response at $1300 \mathrm{~nm}$ of a $200 \mathrm{~m}$-long link for: a DFB optical source with $10 \mathrm{MHz}$ of FWHM; a FP laser of $2 \mathrm{~nm}$ of linewidth; and a LED with $20 \mathrm{~nm}$ of source linewidth. The rest of parameters take the same value as those previously indicated. As expected, the frequency response is progressively penalised as source linewidth increases, hampering the possible observance of high-order resonances. On the other hand, the influence of the launching condition on the frequency response can be seen in Fig. 19(b). A PF GIPOF link of 200m and an input power 
spectral density conforming a Gaussian lineshape from a DFB optical source with $0.2 \mathrm{~nm}$ have been considered. From the frequency response, a dramatic enhancement of the RF baseband bandwidth is observed when applying a RML condition as well as a reduction of the filtering effect, similarly of what it was expected from the silica-based MMF analysis.

(a)

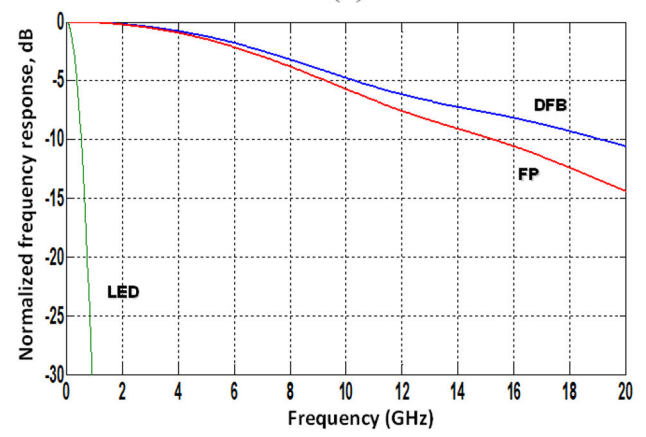

(b)

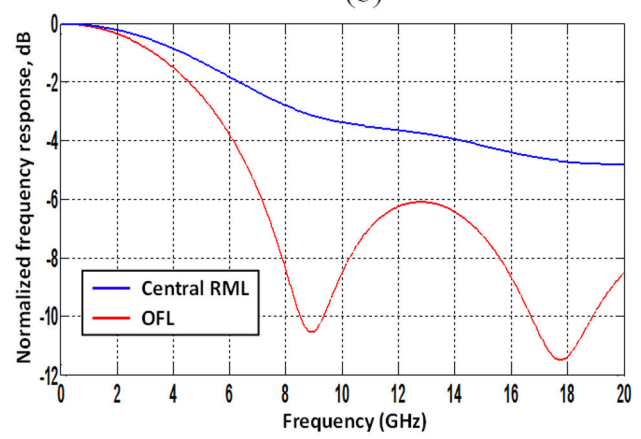

Figure 19. (a) Influence of the optical source temporal coherence on the frequency response of a 200m-long PF GIPOF link. (b) Influence of the light injection on the frequency response of a $200 \mathrm{~m}$-long PF GIPOF link.

Some measurement examples of the PF GIPOF transfer function are presented highlighting the conditions upon broadband transmission in regions far from baseband can be featured thus validating the theoretical model proposed [88]. A comparison between the curves predicted by the theoretical model and the experimental results is also provided. Good agreement between theory and experimental results is observed. The results have been tested over an amorphous perfluorinated (PF) graded-index polymer optical fiber. In all cases, such fiber type fulfils the requirements of the IEC ${ }^{6}$ 60793-2-40 standard for the PF polymerbased POFs (types A4f, A4g and A4h) which fits a minimum bandwidth of 1500MHz@100m for A4f type and 1880Mhz@100m for A4g and A4h types, respectively. The setup schematic for the experimental measurements follows the same concept as that reported in Fig. 13. The experimental results have been calibrated with regards to both the E/O intensity modulator and the photodetector electrical responses. Similar optical sources as those used in silicabased MMFs experiments were employed. An OFL excitation at the fiber input end has been applied. Theoretical curves have been obtained considering a PF GIPOF with a refractive index profile of $\alpha=2.18$ and an intrinsic attenuation coefficient of $\alpha_{0}=42 \mathrm{~dB} / \mathrm{km}$ at $1300 \mathrm{~nm}$. The latter was measured employing Optical Time-Domain Reflectometer (OTDR) techniques. Core and cladding refractive indices have been calculated using a three-term Sellmeier function. It has also been assumed a free chirp source. Differential Mode Attenuation (DMA) effects have been considered by setting $\rho=11 ; \eta=12.2$. Additionally, a random coupling process defined by a Gaussian autocorrelation function has been defined for the mode cou- 
pling with a correlation length of $\varsigma=0.005 \mathrm{~m}$ and $\mathrm{rms}$ deviation of $\varsigma=1.6 \cdot 10^{4} \times \mathrm{a}$, being 'a' the core radius of the fiber considered.

(a)

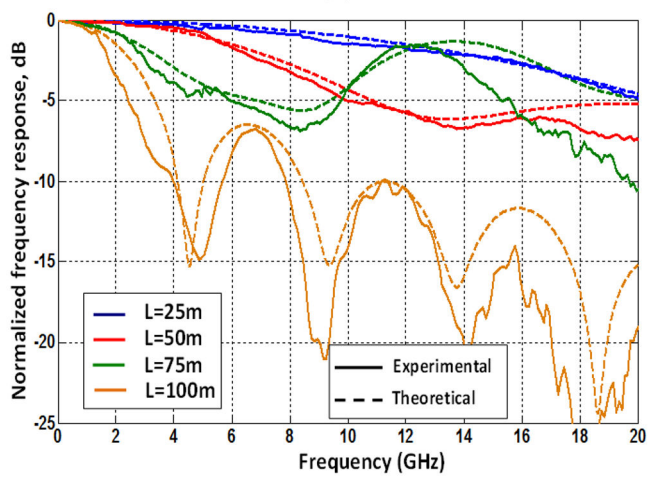

(b)

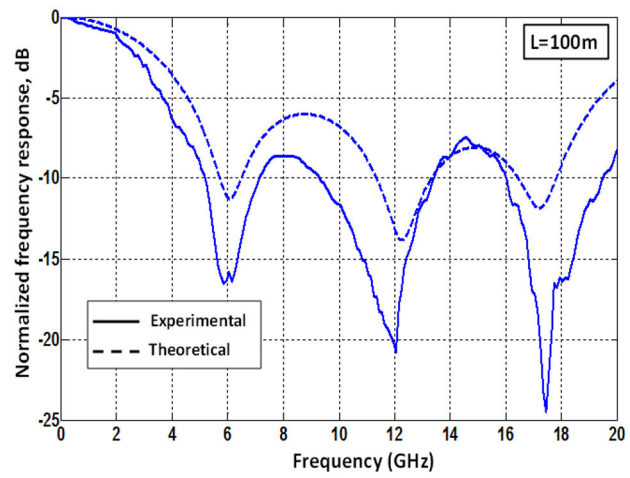

Figure 20. (a) Theoretical and measured frequency response of a 50 $\mu \mathrm{m}$ core diameter PF GIPOF link for different lengths with a FP laser source operating at $1300 \mathrm{~nm}$. (b) Theoretical and measured frequency response of a 100m-long $62.5 \mu \mathrm{m}$ core diameter PF GIPOF link under identical operating conditions.

Fig. 20(a) depicts the theoretical (dashed line) and measured (solid line) frequency responses of a $50 \mu \mathrm{m}$ core diameter PF GIPOF link for different lengths. On the other hand, the theoretical and measured frequency response of a $100 \mathrm{~m}$-long $62.5 \mu \mathrm{m}$ core diameter PF GIPOF link is shown in Fig. 20(b). In both cases, a FP optical source operating at $1300 \mathrm{~nm}$ and $1.8 \mathrm{~nm}$ of linewidth was employed. Results reveal the presence of some latent high-order resonances in the PF GIPOF frequency response. Although these passbands suffer from a power penalty in the range of $5 \mathrm{~dB}$ per passband order, attending to Fig. 20(a), this high attenuation could significantly be improved with lower fiber attenuation values. Nevertheless, the presence of these periodicities in the PF GIPOF frequency response opens up the extension of the transmission capabilities beyond baseband thus increasing the aggregated capacity over this optical fiber type.

Another example can be seen in Fig. 21(a), where the theoretical and measured frequency response of a $150 \mathrm{~m}$-long $120 \mu \mathrm{m}$ core diameter PF GIPOF link is depicted. Similar operating conditions as above were applied. In constrast, Fig. 21(b) reports the RF bandwidth enhancement when employing a narrow-linewidth DFB optical source. A $62.5 \mu \mathrm{m}$ core diameter PF GIPOF was used. As expected, the available bandwidth is increased if we compare the curves within this figure with those obtained in Fig. 20(a). However, it is important to observe that the frequency response at $1550 \mathrm{~nm}$ falls at $17 \mathrm{~dB}$ at $20 \mathrm{GHz}$. This is due to the fact that the PF GIPOF intrinsic attenuation at this wavelength was measured to be $140 \mathrm{~dB} / \mathrm{km}$. In both figures an OFL condition was also applied.

Finally, the following figure evaluates the conditions upon which broadband transmission over PF GIPOF beyond the RF baseband bandwidth is possible. Fig. 22(a) shows the meas- 
(a)

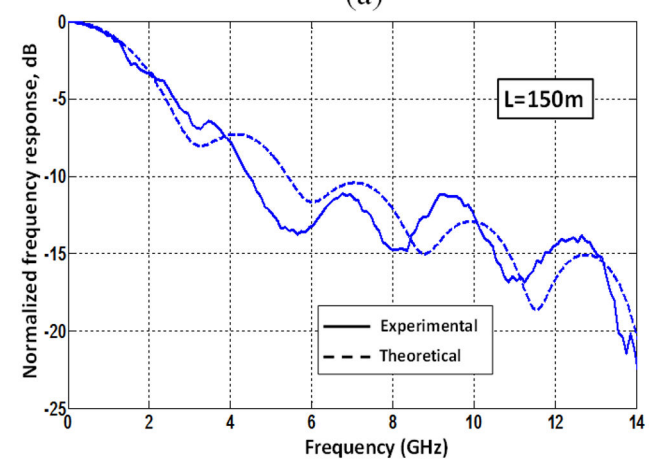

(b)

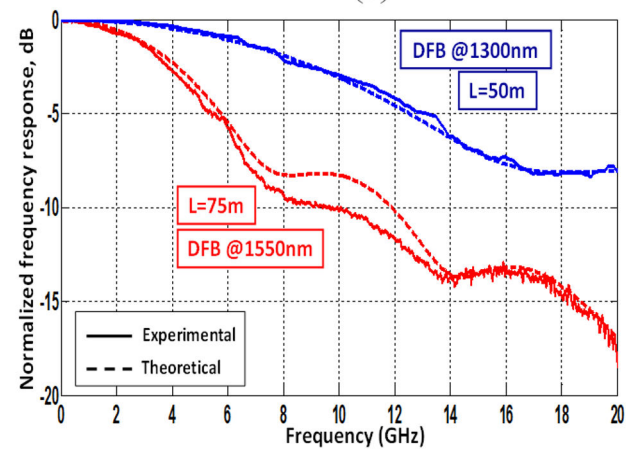

Figure 21. (a) Theoretical and measured frequency response of a 150m-long $120 \mu \mathrm{m}$ core diameter PF GIPOF link, under similar operation conditions as in Fig. 20. (b) Theoretical and measured frequency response of a $62.5 \mu \mathrm{m}$ core diameter PF GIPOF link employing DFB optical sources.

(a)

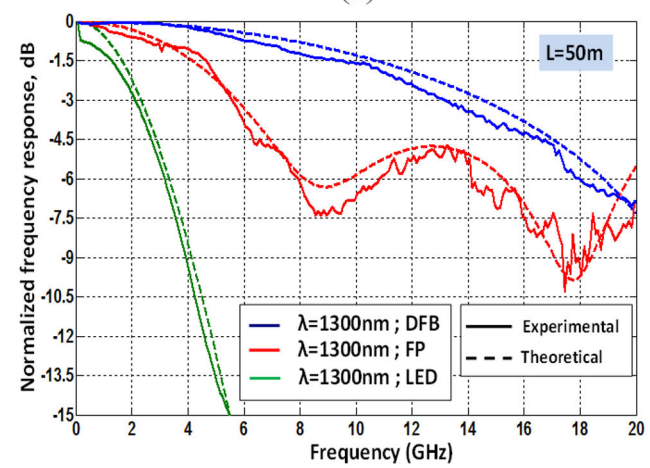

(b)

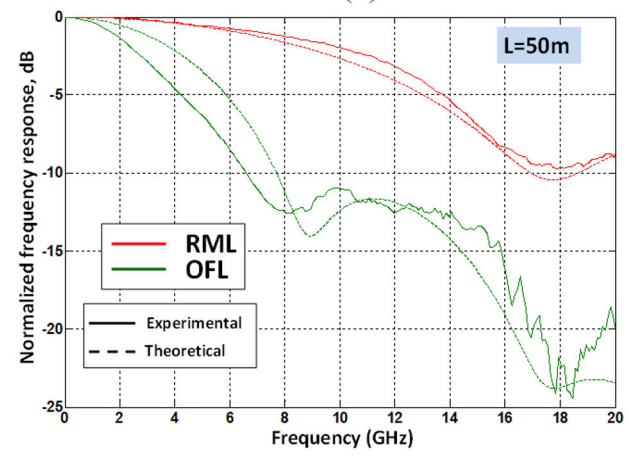

Figure 22. (a) Influence of the optical source temporal coherence on the frequency response of a $50 \mathrm{~m}$-long $62.5 \mu \mathrm{m}$ core diameter PF GIPOF link. (b) Measured influence of the launching condition on the 50m-long $120 \mu \mathrm{m}$ core diameter PF GIPOF frequency response.

ured frequency response for a $50 \mathrm{~m}$-long $62.5 \mu \mathrm{m}$ core diameter PF GIPOF link at an operating wavelength of $1300 \mathrm{~nm}$. As it was expected from the theory, the frequency response dramatically decreases when increasing the rms source linewidth. When a LED with $W=98 \mathrm{~nm}$ of spectral width is employed as the optical source, the frequency response falls after a few GHz. In contrast greater baseband bandwidths when employing a FP laser with $W=1.8 \mathrm{~nm}$ and a DFB source with $100 \mathrm{kHz}$ of FWHM are achievable. In addition, the presence of high-order resonances in the frequency response is also identified. On the other hand, Fig. 22(b) illustrates the frequency response of a 50m-long $120 \mu \mathrm{m}$ core diameter PF GIPOF link at launching conditions OFL and RML, respectively. In both cases a FP laser op- 
erating at $1300 \mathrm{~nm}$ and $1.8 \mathrm{~nm}$ of source linewidth was employed. As expected from theory, RML increases the RF baseband bandwidth as well as flattens the frequency response. However, possible transmission regions beyond baseband are penalised in power due to the high PF GIPOF attenuation compared to that of silica-based counterparts. From both figures, we can therefore conclude, and similarly to silica-based MMFs, that exploiting the possibility of transmitting broadband RF signals in PF GIPOFs at high frequencies is also contingent on the use of narrow-linewidth sources and selective mode-launching schemes.

\section{Conclusions}

Future Internet Access technologies are supposed to bring us a very performing connection to the main door of our homes. At the same tine, new services and devices and their increase use, commonly grouped as next-generation access (NGA) services, will require data transfers at speeds exceeding 1Gbps inside the building or home at the horizon 2015. Both drivers lead to the deployment of a high-quality, future-proof network from the access domain even to inside buildings and homes. There is a widely-spread consensus that FTTx is the most powerful and future-proof access network architecture for providing broadband services to residential users. Furthermore, FTTx deployments with WDM-PON topologies are considered in the long-term the target architecture for the next-generation access networks. This environment may end up taking advantage of optical cabling solutions as an alternative to more traditional copper or pure wireless approaches.

Multimode optical fibers (MMF), both silica- and polymer-based, can offer the physical infrastructure to create a fusion and convergence of the access network via FFTx for these next-generation access (NGA) services. Both fiber types may be used not only to transport fixed data services but also to transparently distribute in-building (and also for short- and medium-reach links) widely ranging signal characteristics of present and future broadband services leading to a significant system-wide cost reduction. The underlying reason is that multimode fibers have a much larger core diameter and thus alignment in fiber splicing and connectorising is more relaxed. Also the injection of light from optical sources is easier, without requiring sophisticated lens coupling systems. And these facts seem to be critical as all optical networks are being deployed even closer to the end users, where most of interconnections are needed. Moreover, polymer optical fiber (POF) may be even easier to install than silica-based multimode fiber, as it is more ductile, easier to splice and to connect even maintaining high bandwidth performances as in the case of PF GIPOFs. However, their main drawback is related to the fact that their bandwidth per unit length is considerably less with respect to singlemode fiber counterparts. However, this may not be decisive as link lengths are relatively short in the user environment.

On the other hand, it is obvious that the deployment of such emerging NGA network technology and its convergence would be not possible without the research and evaluation of predictive and accurate models to describe the signal propagation through both MMF fiber types to overcome the inherent limitations of such a transport information media. However, 
the potentials of MMF to support broadband RF, microwave and millimetre-wave transmission beyond baseband over short and intermediate distances are yet to be fully known, as its frequency response seems to be unpredictable under arbitrary operating conditions as well as fiber characteristics. The different experimental characterizations and the theoretical model presented in this chapter allow understanding and an estimation of the frequency response and the total baseband bandwidth. In addition, can give an estimation of the aggregated transmission capacity over MMFs through analyzing the high-order resonances as well as the presence of relatively flat regions that are present beyond baseband, under certain conditions, in the MMF frequency response.

From the theoretical and experimental results, it is demonstrated that, next to its baseband transmission characteristics, an intrinsically multimode fiber link will show passband characteristics in higher frequency bands. However, the location and the shape of these passbands depend on the actual fiber characteristics, and may change due to environmental conditions and/or the light launching conditions as well as the number of guided modes excited and the power distribution among them. Also the length of the fiber, the mode coupling processes, the source wavelength, the launching scheme, and the fiber core diameter influence the fibre frequency response. This fact imposes a great challenge for the extension of the bandwidth-dependent multimode fiber performance. And, the influence of most of all these parameters that can have a large impact on the date rate transmission performance in MMF links has been addressed. Although no accurate agreement can be expected due to the many approximations made in the theoretical analysis as well as the amount of parameters involved in the frequency response, the results reveal a quite good assessment in the behavior of the multimode optical fiber frequency response compared to the curves predicted by the model

The use of selective mode-launching schemes combined with the use of narrow-linewidth optical sources is demonstrated to enable broadband RF, microwave and millimetre-wave transmission overcoming the typical MMF bandwidth per length product. Under these conditions it is possible to achieve flat regions in the frequency response as well as passband characteristics far from baseband. Transmission of multiple-GHz carrier in these MMF links can be featured at certain frequencies albeit a small power penalty, enabling the extension of broadband transmission, with direct application in Radio-over-Fiber (RoF) systems, in which broadband wireless services could be integrated on the same fiber infrastructure, thereby reducing system costs. The results also reveal that PF GIPOF has some latent highorder passbands and flat regions in its frequency response, which however suffer a high attenuation due to the higher intrinsic attenuation of polymer optical fibers compared to that of silica-based counterparts. Anyway, this power penalty could significantly be improved with lower fiber attenuation values.

To resume, MMFs (both silica- and polymer-based) are still far from SMF bandwidth and attenuation, but they are called to the next step on access network links due to its low cost systems requirements (light sources, optical detectors, larger fiber core,...) against the high cost of the singlemode components. It is worth mentioning that in-building networks may comprise quite a diversity of networks: not only networks within residential homes, but also networks inside office buildings, hospitals, and even more extensive ones such as networks 
in airport departure buildings and shopping malls. Thus the reach of in-building networks may range from less than 100 metres up to a few kilometers. A better understanding of the possibilities of signal transmission outside the baseband of such fibers are investigated, in order to extend their capabilities, together with the evaluation of current fiber frequency response theoretical models becomes of great importance.

\section{Acknowledgements}

The work comprised in this document has been developed in the framework of the activities carried out in the Displays and Photonics Applications group (GDAF) at Carlos III University of Madrid.

This research work has been supported by the following Spanish projects: TEC2006-13273C03-03-MIC, TEC2009-14718-C03-03 and TEC2012-37983-C03-02 of the Spanish Interministerial Comission of Science and Technology (CICyT) and FACTOTEM-CM:S-0505/ESP/000417 and FACTOTEM-2/2010/00068/001 of Comunidad Autónoma de Madrid.

Additional financial support was obtained from the European Networks of Excellence: ePhoton/One+ (FP6-IST-027497) ${ }^{7}$ and and BONE: Building the Future Optical Network in Europe (FP7-ICT-216863) ${ }^{8}$

\section{Author details}

David R. Sánchez Montero and Carmen Vázquez García

Displays and Photonics Applications Group (GDAF), Electronics Technology Dpt., Carlos III University of Madrid, Leganés (Madrid), Spain

\section{References}

[1] Broadband Network Strategies; Strategy Analytics. Global Broadband Forecast: 2H2011 18 Nov 2011.

[2] Charbonnier, B. End-user Future Services in Access, Mobile and In-Building Networks. FP7 ICT-ALPHA project, public deliv. 1.1p, http://www.ict-alpha.eu, July 2008 .

7 ePhoton/One+ is supported by the Sixth Framework Programme (FP6) of the European Union. 8 BONE is supported by the Seventh Framework Programme (FP7) of the European Union. 
[3] Meyer, S. Final usage Scenarios Report. FP7 ICT-OMEGA Project, public deliv. 1.1 Aug. 2008.

[4] Ishigure, T., Aruga, Y. and Koike Y. High-bandwidth PVDF-clad GI POF with ultralow bending loss. Journal of Lightwave Technology 2007; 25(1) 335-345.

[5] Ishigure, T., Makino, K., Tanaka, S. and Koike, Y. High-Bandwidth Graded-Index Polymer Optical Fiber Enabling Power Penalty-Free Gigabit Data Transmission. Journal of Lightwave Technology 2003; 21(11) 2923-2930.

[6] Ziemann, O., Krauser J., Zamzow, P.E. and Daum, W. POF Handbook: Optical Short Range Transmission Systems. Berlin: Springer 2nd Edition; 2008.

[7] Epworth, R.E. The phenomenon of modal noise in analog and digital optical fiber systems. Proceedings of the 4th European Conference on Optical Communications (ECOC), Genova, Italy; 1978.

[8] Bates, R.J.S., Kutcha, D.M. Improved multimode fibre link BER calculations due to modal noise and non-self-pulsating laser diodes. Optical and Quantum Electronics 1995; 27 203-224.

[9] Pepeljugoski, P., Kuchta, D.M. and Risteski, A. Modal noise BER calculations in 10Gb/s multimode fiber LAN links. IEEE Photonics Technology Letters 2005; 17(12) 2586-2588.

[10] Gasulla I. and Capmany, J. Modal noise impact in Radio over Fiber multimode fiber links. Optics Express 2008; 16(1) 121-126.

[11] Daum, W. Krauser, J., Zamzow P. and Ziemann, O. POF - Polymer Optical Fibers for Data Communication. Germany: Springer; 2002.

[12] Li, W., Khoe, G.D., van den Boom, H.P.A., Yabre, G., de Waardt, H., Koike, Y., Yamazaki, S., Nakamura, K. and Kawaharada, Y. 2.5 Gbit/s transmission over $200 \mathrm{~m}$ PPMA graded index polymer optical fiber using a $645 \mathrm{~nm}$ narrow spectrum laser and a silicon APD. Microwave and Optical Technology Letters 1999; 20(3) 163-166.

[13] Groh, W. Overtone absorption in macromolecules for polymer optical fibers. Makromoleculare Chemie 1988; 189 2861-2874.

[14] Ishigure, T., Nihei, E. and Koike, Y. Graded-index polymer optical fiber for highspeed data communication. Applied Optics 1994; 33(19) 4261-4266.

[15] Van den Boom, H.P.A., Li, W., van Bennekom, P.K., Tafur Monroy,I. and Khoe, G.D. High-capacity transmission over polymer optical fiber. IEEE Journal on Selected Topics in Quantum Electronics 2001; 7(3) 461-470.

[16] Nihei, E., Ishigure, T., Taniott, N. and Koike, Y. Present prospect of graded-lndex plastic optical fiber in telecommunication. IEICE Transactions on Electronics 1997; E80-C 117-121. 
[17] Tanio, N. and Koike, Y. What is the most transparent polymer?. Polymer Journal 2000; 32(1) 119-125.

[18] Ishigure, T., Tanaka, S., Kobayashi, E. and Koike, Y. Accurate refractive index profiling in a graded-index plastic optical fiber exceeding gigabit transmission rates. Journal of Lightwave Technology 2002; 20(8) 1449-1456.

[19] Gloge, D. Dispersion in Weakly Guiding Fibers. Applied Optics 1971; 10(11) 2442-2445.

[20] Liu, Y.E., Rahmna, B.M.A., Ning, Y.N. and Grattan, K.T.V. Accurate Mode Characterization of Graded-Index Multimode Fibers for the Application of Mode-Noise Analysis. Applied Optics 1995; 34 1540-1543.

[21] Xiao, J.B. and Sun, X.H. A Modified full-vectorial finite-difference beam propagation method based on H-fields for optical waveguides with step-index profiles. Optics Communications 2006; 266(2) 505-511.

[22] Huang, W.P. and Xu, C.L. Simulation of 3-Dimensional Optical Wave-Guides by a Full-Vector Beam-Propagation Method. IEEE Journal of Quantum Electronics 1993; 29(10) 2639-2649.

[23] Zubia, J., Poisel, H., Bunge, C.A., Aldabaldetreku, G. and Arrue, J. POF Modelling. Proceedings of the 11th International Conference on Plastic Optical Fiber (ICPOF) Tokyo, Japan, 221-224; 2002.

[24] Marcatili, E.A.J. Modal Dispersion in Optical Fibers with Arbitrary Numerical Aperture and Profile Dispersion. Bell Labs Technical Journal 1977; 6(1) 49-63.

[25] Ishigure, T., Nihei, E. and Koike, Y. Optimum refractive-index profile of the gradedindex polymer optical fiber, toward gigabit data links. Applied Optics 1996; 35(12) 2048-2053.

[26] Olshansky, R. and Keck, D.B. Pulse broadening in graded-index optical fibers. Applied Optics 1976; 15 483-491, and errata; Soudagar M.K. and Wali, A.A. Applied Optics 1993; 326678.

[27] Olshansky, R. Mode-Coupling Effects in Graded-Index Optical Fibers. Applied Optics 1975; 14 935-945.

[28] Marcuse, D. Theory of Dielectric Optical Waveguides. New York: Academic Press 2nd Edition; 1991.

[29] Yabre, G. Comprehensive Theory of Dispersion in Graded-Index Optical Fibers. Journal of Lightwave Technology 2000; 18(2) 166-177.

[30] Presby, H.M. and Kaminow, I.P. Binary silica optical fibers: refractive index and profile dispersion measurements. Applied Optics 1976; 15(12) 469-470. 
[31] Olshansky, R. and Nolan, D.A. Mode-dependent attenuation of optical fibers: Excess Loss. Applied Optics 1976; 15 1045-1047.

[32] Gloge, D. Weakly Guiding Fibers. Applied Optics 1971; 10(10) 2252-2258.

[33] Yabre, G. Theoretical investigation on the dispersion of graded-index polymer optical fibers. Journal of Lightwave Technology 2000; 18(6) 869-877.

[34] Mickelson, A.R. and Eriksrud, M. Mode-dependent attenuation in optical fibers. Journal of the Optical Society of America 1983; 73(10) 1282-1290.

[35] Golowich, S.E., White, W., Reed, W.A. and Knudsen, E. Quantitative estimates of mode coupling and differential modal attenuation in perfluorinated graded-index plastic optical fiber. Journal of Lightwave Technology 2003; 21(1) 111-121.

[36] White, W.R., Dueser, M., Reed, W.A. and Onishi, T. Intermodal dispersion and mode coupling in perfluorinated graded-index plastic optical fiber. IEEE Photonics Technology Letters 1999; 11(8) 997-999.

[37] Cancellieri, G. Mode-Coupling in Graded-Index Optical Fibers Due to Perturbation of the Index Profile. Applied Physics 1980; 23 99-105.

[38] Cancellieri, G. Mode-Coupling in Graded-Index Optical Fibers Due to Micro-Bending. Applied Physics and Materials Science \& Processing 1981; 26 51-57.

[39] Su, L., Chiang, K.S. and Lu, C. Microbend-induced mode coupling in a graded-index multimode fiber. Applied Optics 2005; 44(34) 7394-7402.

[40] Ohashi, M., Kitayama, K. and Seikai, S. Mode-Coupling Effects in a Graded-Index Fiber Cable. Applied Optics 1981; 20 2433-2438.

[41] Djordjevich A. and Savovic, S. Investigation of mode coupling in step index plastic optical fibers using the power flow equation. IEEE Photonics Technology Letters 2000; 12(11) 1489-1491.

[42] Donlagic, D. Opportunities to enhance multimode fiber links by application of overfilled launch. Journal of Lightwave Technology 2005; 23(11) 3526-3540.

[43] Ishigure, T., Ohdoko, K., Ishiyama, Y. and Koike, Y. Mode-coupling control and new index profile of GI POF for restricted-launch condition in very-short-reach networks. Journal of Lightwave Technology 2005; 23(12) 4155-4168.

[44] Polley, A. High Performance Multimode Fiber Systems: A Comprehensive Approach. PhD Thesis. Georgia Institute of Technology; December 2008.

[45] Cunningham, D.G., Lane, W.G. and LAne, B. Gigabit Ethernet Networking. Indianapolis: Macmillan; 1999.

[46] Gasulla I. and Capmany, J. 1 Tb/s km Multimode fiber link combining WDM transmission and low-linewidth lasers. Optics Express 2008; 16(11) 8033-8038. 
[47] Sim, D.H., Takushima, Y. and Chung Y.C. High-Speed Multimode Fiber Transmission by Using Mode-Field Matched Center-Launching Technique. Journal of Lightwave Technology 2009; 27(8) 1018-1026.

[48] Zeng, J., Lee, S.C.J., Breyer, F., Randel, S., Yang, Y., van den Boom, H.P.A. and Koonen, A.M.J. Transmission of $1.25 \mathrm{~Gb} / \mathrm{s}$ per Channel over 4.4. $\mathrm{km}$ Silica Multimode Fibre Using QAM Subcarrier Multiplexing. Proceedings of the 33th European Conference on Optical Communications (ECOC), paper 7.4.3, Berlin, Germany; 2007.

[49] WiMedia Alliance. Worlwide regulatory status. http://www.wimedia.org.

[50] Koonen, A.M.J. and Garcia Larrode, M. Radio-Over-MMF Techniques, Part II: Microwave to Millimeter-Wave Systems. Journal of Lightwave Technology 2008; 26(15) 2396-2408.

[51] Lee, S.C.J., Breyer, F., Randel, S., Spinnler, B., Polo, I.L.L., van Den Borne, D., Zeng, J., de Man, E., van Den Boom, H.P.A. and Koonen, A.M.J. 10.7 Gbit/s transmission over $220 \mathrm{~m}$ polymer optical fiber using maximum likelihood sequence estimation. Proceedings of Optical Fiber Communication and the National Fiber Optic Engineers Conference (OFC/NFOEC), Anaheim, USA; 2007.

[52] Polley, A. and Ralph, S.E. 100 m, 40 Gb/s Plastic Optical Fiber Link. Proceedings of Optical Fiber Communication and the National Fiber Optic Engineers Conference (OFC/NFOEC), San Diego, USA; 2008.

[53] Yang, H., Lee, S.C.J., Tangdiongga, E., Okonkwo, C., van den Boom, H.P.A., Breyer, F., Randel, S. and Koonen, A.M.J. 47.4 Gb/s Transmission Over 100 m Graded-Index Plastic Optical Fiber Based on Rate-Adaptive Discrete Multitone Modulation. Journal of Lightwave Technology 2010; vol. 28(4) 352-359.

[54] Montero, D.S. Multimode Fiber Broadband Access and Self-Referencing Sensor Networks. PhD Thesis. Electronics Technlogy Dpt., Universidad Carlos III de Madrid, Leganés (Madrid), Spain; 2011.

[55] Ingham, J.D., Webster, M., Wake, D., Seeds, A.J., Penty, R.V. and White, I.H. Bidirectional Transmission of 32-QAM Radio Over a Single Multimode Fibre Using 850-nm Vertical-Cavity Half-Duplex Transceivers. Proceedings of the 28th European Conference on Optical Communications (ECOC), Copenhagen, Denmark; 2002.

[56] Gasulla I. and Capmany, J. Simultaneous baseband and radio over fiber signal transmission over a $5 \mathrm{~km}$ MMF link. Proceedings of International Topics Meeting on Microwave Photonics Conference (MWP), pp. 209-212, Gold Coast, USA; 2008.

[57] Garcia Larrode, M., Koonen, A.M.J. and Vegas Olmos J.J. Overcoming Modal Bandwidth Limitation in Radio-over-Multimode Fiber Links. IEEE Photonics Technology Letters 2006; 18(22) 2428-2430. 
[58] Garcia Larrode, M. and Koonen, A.M.J. Theoretical and Experimental Demonstration of OFM Robustness Against Modal Dispersion Impairments in Radio Over Multimode Fiber Links. Journal of Lightwave Technology 2008; 26(12) 1722-1728.

[59] Garcia Larrode, M., Koonen, A.M.J. and Vegas Olmos, J.J. Fiber-based broadband wireless access employing optical frequency multiplication. IEEE Journal of Selected Topics in Quantum Electronics 2006; 12(4) 875-881.

[60] Garcia Larrode, M., Koonen, A.M.J., Vegas Olmos, J.J. and Ng'Oma, A. Bidirectional radio-over-fiber link employing optical frequency multiplication. IEEE Photonics Technology Letters 2006; 18(1) 241-243.

[61] Raddatz L. and White, I.H. Overcoming the modal bandwidth limitation of multimode fiber by using passband modulation. IEEE Photonics Technology Letters 1999; 11(2) 266-268.

[62] Tyler, E.J., Webster, M., Penty, R.V. and White, I.H. Penalty free subcarrier modulated multimode fiber links for datacomm applications beyond the bandwidth limit. IEEE Photonics Technology Letters 2002; 14(1) 110-112.

[63] Zhao, X. and Choa, F.S. Demonstration of $10-\mathrm{Gb} / \mathrm{s}$ transmissions over a $1.5-\mathrm{km}-\mathrm{long}$ multimode fiber using equalization techniques. IEEE Photonics Technology Letters 2002; 14(8) 1187-1189.

[64] Pepeljugoski, P., Schaub, J., Tierno, J., Wilson, B., Kash, J., Gowda, S., Wu, H. and Hajimiri, A. Improved Performance of $10 \mathrm{~Gb} / \mathrm{s}$ Multimode Fiber Optic Links Using Equalization. Proceedings of Optical Fiber Communication and the National Fiber Optic Engineers Conference (OFC/NFOEC), pp. 472-474, Atlanta, USA; 2003.

[65] Raddatz, L., White, I.H., Cunningham, D.G., Nowell, M.C, Tan, M.R.T. and Wang, S.Y. Fiber-optic m-ary modulation scheme using multiple light sources. Proceedings of Optical Fiber Communication and the National Fiber Optic Engineers Conference (OFC/NFOEC), pp. 198-199, Dallas, USA; 1997.

[66] Raddatz, L., White, I.H., Cunningham, D.G. and Nowell, M.C. An experimental and theoretical study of the offset launch technique for the enhancement of the bandwidth of multimode fiber links. Journal of Lightwave Technology 1998; 16(3) 324-331.

[67] Webster, M., Raddatz, L., White, I.H. and Cunningham, D.G. A statistical analysis of conditioned launch for gigabit ethernet links using multimode fiber. Journal of Lightwave Technology 1999; 17(9) 1532-1541.

[68] Sun, Q., Ingham, J.D., Penty, R.V., White, I.H. and Cunningham, D.G. Twin-Spot Launch for Enhancement of Multimode-Fiber Communication Links. Proceedings of Conference on Lasers and Electro-Optics CLEO; Baltimore, USA; 2007.

[69] Stuart, H.R. Dispersive multiplexing in multimode fiber. Science 2000; 289 305-307.

[70] De Boer, M., Tsekrekos, C.P., Martinez, A., Kurniawan, H., Bergmans, J.W.H, Koonen, A.M.J., van den Boom, H.P.A. and Willems, F.M.J. A first demonstrator for a 
mode group diversity multiplexing communication system. Proceeding of IEE Seminar on Optical Fibre Communications and Electronic Signal Processing, pp. 0_46-16/5, London, UK; 2005.

[71] Dixon, B.J., Pollard, R.D. and Iezekiel, S. Orthogonal frequency-division multiplexing in wireless communication systems with multimode fiber feeds. IEEE Transactions on Microwave Theory and Techniques 2001; 49(8) 1404-1409.

[72] Shen, X., Kahn, J.M. and Horowitz, M.A. Compensation for multimode fiber dispersion by adaptive optics. Optics Letters 2005; 30(22) 2985-2987.

[73] Patel, K.M., Polley, A., Balemarthy, K. and Ralph, S.E. Spatially resolved detection and equalization of modal dispersion limited multimode fiber links. Journal of Lightwave Technology 2006; 24(7) 2629-2636.

[74] Gu, X.J., Mohammed, W. and Smith, P.W. Demonstration of all-fiber WDM for multimode fiber local area networks. IEEE Photonics Technology Letters 2006; 18(1) 244-246.

[75] Yadlowsky M.J. and Mickelson, A.R. Distributed loss and mode coupling and their effect on time-dependent propagation in multimode fibers. Applied Optics 1993; 32(33) 6664-6677.

[76] Gloge, D. Optical power flow in multimode optical fibers. Bell Labs Technical Journal 1972; 51 1767-1783.

[77] Tatekura, K, Itoh, K. and Matsumoto, T. Techniques and Formulations for Mode Coupling of Multimode Optical Fibers. IEEE Transactions on Microwave Theory and Techniques 1978; 26(7) 487-493.

[78] Djordjevich, A. and Savovic. S. Numerical solution of the power flow equation in step-index plastic optical fibers. Journal of the Optical Society of America: B-Optical Physics 2004; 21 1437-1442.

[79] Zubia, J., Durana, G., Aldabaldetreku, G., Arrue, J., Losada, M.A. and Lopez-Higuera, M. New method to calculate mode conversion coefficients in SI multimode optical fibers. Journal of Lightwave Technology 2003; 21(3) 776-781.

[80] Stepniak, G. and Siuzdak, J. An efficient method for calculation of the MM fiber frequency response in the presence of mode coupling. Optical and Quantum Electronics 2006; 38(15) 1195-1201.

[81] Saleh, B.E.A. and Abdula, R.M. Optical Interference and Pulse-Propagation in Multimode Fibers. Fiber and Integrated Optics 1985; 5 161-201.

[82] Gasulla, I. and Capmany, J. Transfer function of multimode fiber links using an electric field propagation model: Application to Radio over Fibre Systems. Optics Express $2006 ; 14(20) 9051-9070$. 
[83] Capmany, J., Ortega, B., Pastor, D. and Sales, S. Discrete-Time Optical Signal Processing of Microwave Signals. Journal of Lightwave Technology 2005; 23(2) 703-723.

[84] Montero, D.S. and Vázquez, C. Temperature impairment characterization in radioover-multimode fiber systems. Optical Engineering 2012; 51(8) 085001-7.

[85] Hackert, M.J. Characterizing multimode fiber bandwidth for Gigabit Ethernet applications. Proceedings of Symposium on Optical Fiber Measurements 1998; 113-118.

[86] TIA/EIA Fiber Optic Test Procedure (FOTP) 54. Mode Scrambler Requirements for overfilled Launching Conditions to Multi-Mode Fibers; Dec 2001.

[87] Ishigure, T., Sato, M., Kondo, A. and Koike, Y. High-bandwidth graded-index polymer optical fiber with high-temperature stability. Journal of Lightwave Technology 2002; 20(8) 1443-1448.

[88] Montero, D.S. and Vázquez, C. Analysis of the electric field propagation method: theoretical model applied to perfluorinated graded-index polymer optical fiber links. Optics Letters 2011; 36(20) 4116-4118. 
